TRANSACTIONS OF THE

AMERICAN MATHEMATICAL SOCIETY

Volume 365, Number 12, December 2013, Pages 6517-6541

S 0002-9947(2013)05892-8

Article electronically published on May 30, 2013

\title{
ON THE TEICHMÜLLER THEORY OF CIRCLE PATTERNS
}

\author{
ZHENGXU HE AND JINSONG LIU
}

\begin{abstract}
Given a circle pattern on the Riemann sphere $\hat{\mathbb{C}}$, in this paper we prove that its quasiconformal deformation space can be naturally identified with the product of the Teichmüller spaces of its interstices.
\end{abstract}

\section{INTRODUCTION}

In this paper we consider the characterization problem of circle patterns in the Riemann sphere $\hat{\mathbb{C}}$. A finite circle pattern $P$ on $\hat{\mathbb{C}}$ is defined to be a collection of circles in $\hat{\mathbb{C}}$ in which no circle has its interior contained in the union of interiors of two other circles. The contact graph $G=G_{P}$ of such a pattern $P$ is a graph whose vertices correspond to the circles in the pattern, and an edge appears in $G$ if and only if the corresponding circles intersect each other. Please refer to [2, 6, 14, 19] for the basic notation on circle patterns (or circle packings).

Let $V$ denote the set of vertices in the contact graph $G=G_{P}$, and let $E$ denote the set of edges in $G$. Sometimes we denote it by $G=(V, E)$. For any vertex $v \in V$, let $P(v)$ be the circle in the pattern $P$ associated with the vertex $v$. For any edge $[v, w] \in E$, the dihedral angle $\Theta_{P}([v, w])$ of the pair of intersecting circles $P(v)$ and $P(w)$ is defined to be the angle in $[0, \pi)$ between the clockwise tangent of $P(v)$ and the counterclockwise tangent of $P(w)$ at a point of $P(v) \cap P(w)$. The function $\Theta_{P}: E \rightarrow[0, \pi)$ is called the dihedral angle function of $P$; see Figure 1.

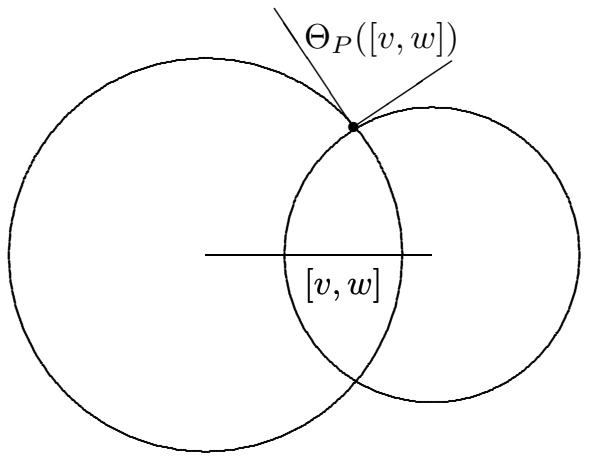

Figure 1

For notational simplicity, in this paper we only consider those circle patterns $P$ with $0 \leq \Theta_{P} \leq \pi / 2$. The more general case, when $0 \leq \Theta<\pi$, is technically

Received by the editors October 13, 2011 and, in revised form, May 31, 2012.

2010 Mathematics Subject Classification. Primary 30C35, 30C80.

The second author was supported by the National Natural Science Foundation of China (Grant No. 10831004). 
more complicated and it will be considered in a future paper. Also in that paper we will combine the idea of a 'polar set' to generalize a characterization of convex polyhedra in hyperbolic 3-space, due to I. Rivin and C. Hodgson [13].

For any vertex $v \in G_{P}$, we denote by $A_{v}$ the Euclidean center of the circle $P(v)$. Then $A_{v}$ lies outside of any other circles of $P$. For any edge $[v, w] \in E$, the straight $\operatorname{arc} A_{v} A_{w}$ is disjoint from the interior of other circles $P(u)$ with $u \neq v, w$. In particular the natural map which maps an edge $[v, w] \in E$ homeomorphically onto the arc $A_{v} A_{w} \subset \hat{\mathbb{C}}$ is an immersion of the contact graph $G_{P}$ into $\hat{\mathbb{C}}$. The only possible double points of this immersion is described in Figure 2.

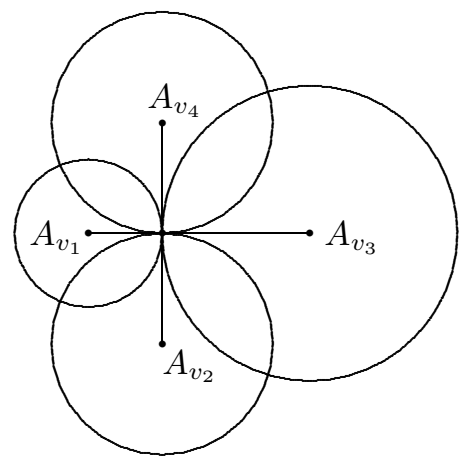

(a)

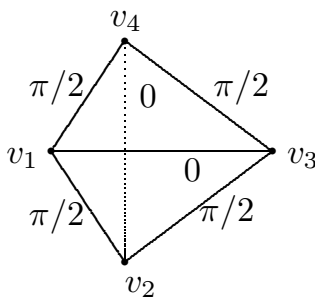

(b)

Figure 2. (a) The configuration of the circles $P\left(v_{l}\right), 1 \leq l \leq 4$.

(b) The corresponding contact graph with dihedral angles marked on the edges.

This special case can be described as follows: the dihedral angles

$$
\Theta_{P}\left(\left[v_{l}, v_{l+1}\right]\right)=\pi / 2, \quad 1 \leq l \leq 4,
$$

where $v_{5} \equiv v_{1}$ and either $\Theta_{P}\left(\left[v_{1}, v_{3}\right]\right)$ or $\Theta_{P}\left(\left[v_{2}, v_{4}\right]\right)$ is equal to 0 . In this case we can easily show

$$
\Theta_{P}\left(\left[v_{1}, v_{3}\right]\right)=\Theta_{P}\left(\left[v_{2}, v_{4}\right]\right)=0 .
$$

Obviously the straight $\operatorname{arc} A_{v_{1}} A_{v_{3}}$ intersects the straight arc $A_{v_{2}} A_{v_{4}}$. In this case the edges $\left[v_{1}, v_{3}\right]$ and $\left[v_{2}, v_{4}\right]$ in $G_{P}$ will be called reducible. Then the reduced contact graph, consisting of the same vertex set $V$ and the irreducible edges in $E$, is embedded in $\hat{\mathbb{C}}$.

If a simple loop $v_{1}, v_{2}, v_{3}, v_{4}, v_{5} \equiv v_{1}$ in a graph $G$ has the property that $\Theta\left(\left[v_{l}, v_{l+1}\right]\right)=\pi / 2, l=1,2,3,4$, and $\Theta\left(\left[v_{1}, v_{3}\right]\right)=0$ as in Figure 2, then let $\tilde{G}$ denote the graph obtained by adding the edge $\left[v_{2}, v_{4}\right]$ to the graph $G$. Define a new function $\tilde{\Theta}$ by letting $\tilde{\Theta}(e)=\Theta(e)$ if $e$ is an edge in $G$, and $\tilde{\Theta}(e)=0$ if $e$ is in $\tilde{G} \backslash G$. A circle pattern $P$ is said to realize the data $(G, \Theta)$ if its contact graph is combinatorially isomorphic to $\tilde{G}$ and the corresponding dihedral angle function is equal to $\tilde{\Theta}$.

Before giving the problems, let us introduce some notation. 
For any abstract topological graph $G$, a map $\phi: G \rightarrow \hat{\mathbb{C}}$ is called an embedding if it is a homeomorphism onto its image. Two embedding spaces $\phi_{0}: G \rightarrow \hat{\mathbb{C}}$ and $\phi_{1}: G \rightarrow \hat{\mathbb{C}}$ are called isotopic, if there exists a continuous map

$$
\Phi: G \times[0,1] \rightarrow \hat{\mathbb{C}},
$$

such that for each $t \in[0,1]$, the map $\Phi_{t} \equiv \Phi(\cdot, t): G \rightarrow \hat{\mathbb{C}}$ is an embedding and $\Phi_{0}=\phi_{0}, \Phi_{1}=\phi_{1}$. An embedded graph in $\hat{\mathbb{C}}$ means an abstract graph $G$ together with an embedding into the Riemann sphere $\hat{\mathbb{C}}$. Two embedded graphs in $\hat{\mathbb{C}}$ with the same vertex set $V$ will be called isomorphic, if they are equal as abstract graphs, and their embeddings are isotopic in $\hat{\mathbb{C}}$.

Note that the graphs $G_{1}, G_{2}$ in Figure 3 are equivalent as abstract graphs, but they are not equivalent as embedded graphs in $\hat{\mathbb{C}}$.

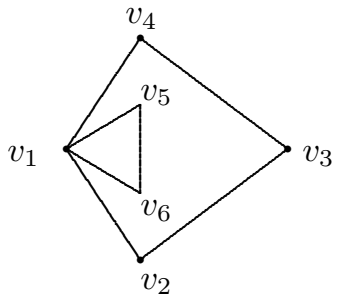

$G_{1}$

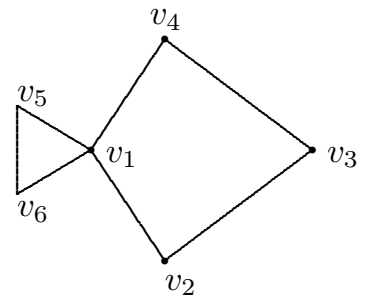

$G_{2}$

FIGURE 3

From now on an embedded contact graph will have no self-loops or double edges. Also, we only consider connected contact graphs of circle patterns. That means, the union of circles in a circle pattern $P$ is a connected set. The non-connected case is essentially almost the same and technically more involved. We omit it here.

Let $P, Q$ be two finite circle patterns on $\hat{\mathbb{C}}$. The patterns $P, Q$ are called equivalent if and only if there exists a Mobiüs transformation $T: \hat{\mathbb{C}} \rightarrow \hat{\mathbb{C}}$ such that $T(P)=Q$. Of course, if $P, Q$ are equivalent, then the contact graph $G_{P}$ is obviously isomorphic to $G_{Q}$ as embedded graphs, and their dihedral angle functions $\Theta_{P}=\Theta_{Q}$. One of the major problems concerning circle patterns is to find necessary and sufficient conditions for two circle patterns to be Möbius equivalent.

Suppose that $G=(V, E)$ is an embedded graph in the Riemann sphere and let $\Theta: E \rightarrow[0, \pi / 2]$ be a function defined on the edges. From now on we fix a circle pattern $P$ which realizes the data $(G, \Theta)$.

A subset of $G$ is said to separate the vertices set $V$, if there is a pair of vertices in the complement of this subset so that any path joining the vertices passes through this subset. Then the dihedral angle function $\Theta_{P}: E \rightarrow[0, \pi / 2]$ of $P$ must satisfy the following conditions; please refer to [6, 19].

(i) If a simple loop in $G$ formed by three edges $e_{1}, e_{2}, e_{3}$ separates the vertices set $V$, then

$$
\sum_{l=1}^{3} \Theta_{P}\left(e_{l}\right)<\pi .
$$


(ii) If $v_{1}, v_{2}, v_{3}, v_{4}, v_{5} \equiv v_{1}$ are distinct vertices in $G$ and if $\left[v_{l}, v_{l+1}\right] \in E$ and $\Theta_{P}\left(\left[v_{l}, v_{l+1}\right]\right)=\pi / 2,1 \leq l \leq 4$, then either $\left[v_{1}, v_{3}\right]$ or $\left[v_{2}, v_{4}\right]$ is an edge in $G$.

Using $V$ as the 0 -skeleton and $E$ as the 1-skeleton, we obtain several faces in $\hat{\mathbb{C}}$, denoted by $\left\{\mathcal{F}_{1}, \mathcal{F}_{2}, \cdots, \mathcal{F}_{p}\right\}$. For our purposes we always assume in this paper that $(G, \Theta)$ satisfies the following additional condition:

(iii) Either there are no triangles in the face set $\left\{\mathcal{F}_{1}, \mathcal{F}_{2}, \cdots, \mathcal{F}_{p}\right\}$ or there is a triangle in $\left\{\mathcal{F}_{1}, \mathcal{F}_{2}, \cdots, \mathcal{F}_{p}\right\}$ whose boundary edges $\left\{e_{1}, e_{2}, e_{3}\right\}$ satisfy $\sum_{l=1}^{3} \Theta\left(e_{l}\right)<$ $\pi$.

Remark 1.1. If the embedded graph $G$ is isomorphic to the skeleton of a triangulation of $\hat{\mathbb{C}}$ with at least five vertices, and its dihedral angle function satisfies (i), (ii), (iii), by using Thurston's interpretation of Andreev's theorem and a compactness argument, we may show that there exists a circle pattern on $\hat{\mathbb{C}}$ realizing the data $(G, \Theta)$; see e.g. 6, 12, 19]. If the $G$ is not a triangulation of $\hat{\mathbb{C}}$, then we may also show that there exists a circle pattern on $\hat{\mathbb{C}}$ realizing $(G, \Theta)$. Please refer to the realization part of Section 3.4

In this paper we shall study the realization problem when the embedded graph $G$ is not isomorphic to the skeleton of a triangulation of $\hat{\mathbb{C}}$ and the dihedral angle function satisfies conditions $(i),(i i)$ and $(i i i)$.

Recall that $P$ is the fixed circle pattern with contact graph $G=(V, E)$. For $v \in$ $V$, denote by $D(v)$ the open disk bounded by the circle $P(v)$. For any component $I$ of $\hat{\mathbb{C}}-\bigcup_{v \in V} D(v)$, since the circle pattern $P$ is finite, the region $I$ has only finitely many boundary components; each boundary component is a piecewise smooth curve formed by finitely many circular arcs or circles. Each (maximal) circular arc or circle on the boundary $\partial I$ belongs to a unique circle in $P$, and therefore is marked by an element of $V$. The region $I$, together with a marking of the circular arcs or circles on its boundary by elements of $V$ is called an interstice of $P$.

For any interstice $I$ of $P$, two quasiconformal mapping: ${ }_{1}^{1} h_{1}, h_{2}: I \rightarrow \hat{\mathbb{C}}$ are called equivalent, if $h_{2} \circ\left(h_{1}\right)^{-1}: h_{1}(I) \rightarrow h_{2}(I)$ is isotopic to a conformal homeomorphism $g$ such that for each circular arc or circle $\gamma \subset \partial I$, the homeomorphism $g$ maps $h_{1}(\gamma)$ onto $h_{2}(\gamma)$.

Definition 1.2. The Teichmüller space of $I$, denoted by $\mathcal{T}_{I}$, is the space of all equivalence classes of quasiconformal mappings $h: I \rightarrow \hat{\mathbb{C}}$.

If the interstice $I$ is a $k$-sided polygon, it follows from the classical Teichmüller theory that $\mathcal{T}_{I}$ is diffeomorphic to the Euclidean space $\mathbb{R}^{k-3}$; see e.g. 11, 10, 11.

The main result of this paper is the following theorem.

Theorem 1.3. Assume $G$ is a graph, not a tetrahedron, embedded in $\hat{\mathbb{C}}$ and assume $\Theta: E \rightarrow[0, \pi / 2]$ is a dihedral angle function for $G$ satisfying conditions $(i),(i i)$ and (iii). Then the space of equivalence classes of circle patterns realizing $(G, \Theta)$ can be naturally identified with the product of the Teichmuller spaces $\Pi_{1}^{p} \mathcal{T}_{I_{i}}$, where $\left\{I_{1}, I_{2}, \cdots, I_{p}\right\}$ are interstices of $P$.

\footnotetext{
${ }^{1}$ An orientation preserving map $f: U \rightarrow W$ between two regions in $\mathbb{C}$ is quasiconformal if and only if, for some $K \geq 1$,

$$
\limsup _{r \rightarrow 0^{+}} \frac{\max _{|z-\zeta|=r}|f(z)-f(\zeta)|}{\min _{|z-\zeta|=r}|f(z)-f(\zeta)|} \leq K, \quad \zeta \in U .
$$
}

We refer to [10, 11] for general background on quasiconformal mappings. 
Note the Teichmüller space of a 3-sided polygon consists of a single point. Hence we have the following corollary.

Corollary 1.4. Let $G=(V, E)$ be the 1-skeleton of a triangulation of $\hat{\mathbb{C}}$ which satisfies $(i)$, (ii) and (iii). If $P=\{P(v): v \in V\}$ and $Q=\{Q(v): v \in V\}$ are circle patterns in $\hat{\mathbb{C}}$ with the same contact graph $G$ such that $\Theta_{P}=\Theta_{Q}: E \rightarrow[0, \pi / 2]$, then $P, Q$ are Mobiüs equivalent.

Remark 1.5. The corollary is weaker than the uniqueness in the Andreev-Thurston result, which does not assume condition ( $i i i)$. Also the Marden-Rodin version of this result assumes (iii); please refer to [12].

Notational conventions. Through this paper, for any round circle $c$ in the complex plane, we denote by $\rho(c)$ its Euclidean radius. For any finite set $A$, we denote by $|A|$ the number of elements in $A$.

\section{Proof of the MAIN THEOREM}

Observe that $P$ is the circle pattern with embedded graph $G=(V, E)$ and dihedral function $\Theta: E \rightarrow[0, \pi / 2]$. If $Q=\{Q(v): v \in V\}$ is another circle pattern with contact graph $G$ and $\Theta_{P}=\Theta_{Q}$, then there is a natural one-to-one correspondence between their interstices; each interstice of $Q$ is quasiconformally equivalent to the corresponding interstice of $P$.

Denote by $\mathcal{D}_{P}$ the equivalent class space of quasiconformal deformations of $P$. Then we have the following.

Lemma 2.1. The space $\mathcal{D}_{P}$ is homeomorphic to an open real k-dimensional manifold, where $k=3 \cdot|V|-|E|-6$.

Proof. For each $v \in V$, the center of the circle $Q(v)$ in a circle pattern $Q \in \mathcal{D}_{P}$ is determined by three real numbers $(x, y, r)$, where $(x, y)$ is its coordinate and $r>0$ is its radius. Each value $\Theta(e), e \in E$, gives one restriction to the parameter system of the deformation space $\mathcal{D}_{P}$.

Note that the dimension of the Mobiüs transformation group is 6 . Therefore $\mathcal{D}_{P}$ is homeomorphic to an open real $k$-dimensional manifold, where $k=3 \cdot|V|-|E|-$ 6 .

Proof of Theorem 1.3. Recall that $\left\{I_{1}, I_{2}, \cdots, I_{p}\right\}$ is the set of interstices of $P$. For any circle pattern $\tilde{P} \in \mathcal{D}_{P}$, we denote by $\left\{\tilde{I}_{1}, \tilde{I}_{2}, \cdots, \tilde{I}_{p}\right\}$ the corresponding interstices. For each interstice $I_{i}, 1 \leq i \leq p$, there is a quasiconformal map $\tau_{i}$ : $I_{i} \rightarrow \tilde{I}_{i} \subset \hat{\mathbb{C}}$ which maps a circle or circular arc on the boundary $\partial I_{i}$ onto a circle or circular $\operatorname{arc}$ on $\partial \tilde{I}_{i}$. Then we have a natural map

$$
H: \mathcal{D}_{P} \rightarrow \prod_{i=1}^{p} \mathcal{T}_{I_{i}}
$$

by sending $\tilde{P}$ to $\left(\left[\tau_{1}\right],\left[\tau_{2}\right], \cdots,\left[\tau_{p}\right]\right) \in \prod_{i=1}^{p} \mathcal{T}_{I_{i}}$, where $\mathcal{T}_{I_{i}}$ is the Teichmüller space of $I_{i}$. Obviously $H$ is well defined.

Denote by $\Gamma$ the faces set $\left\{\mathcal{F}_{1}, \mathcal{F}_{2}, \cdots, \mathcal{F}_{p}\right\}$ and denote by $F$ the set of interstices $\left\{I_{1}, I_{2}, \cdots, I_{p}\right\}$ of $P$. Then $p=|F|=|\Gamma|$. When $G$ is connected, each face in $\Gamma$ is simply connected. From Euler's formula, it follows that

$$
|V|-|E|+|\Gamma|=2 \text {. }
$$


On the other hand,

$$
\operatorname{dim}_{\mathbb{R}} \prod_{i=1}^{p} \mathcal{T}_{I_{i}}=\sum_{i=1}^{p} \operatorname{dim}_{\mathbb{R}} \mathcal{T}_{I_{i}}=\sum_{i=1}^{p}\left(\left|E_{i}\right|-3\right)=\sum_{i=1}^{p}\left(\left|\mathcal{E}_{i}\right|-3\right)=\sum_{i=1}^{p}\left|\mathcal{E}_{i}\right|-3 \cdot|\Gamma|,
$$

where $E_{i}$ is the edge set of the interstice $I_{i}$ and $\mathcal{E}_{i}$ is the edge set of the face $\mathcal{F}_{i}, 1 \leq i \leq p$. Since each edge in $E$ is the common boundary of two faces in $\Gamma$ (maybe the same), we deduce that

$$
\operatorname{dim}_{\mathbb{R}}\left(\prod_{i=1}^{p} \mathcal{T}_{I_{i}}\right)=2 \cdot|E|-3 \cdot|\Gamma|=3 \cdot|V|-|E|-6 .
$$

The continuity of $H: \mathcal{D}_{P} \rightarrow \prod_{i=1}^{p} \mathcal{T}_{I_{i}}$ follows from basic quasiconformal mapping theory; please refer to [11.

The proofs of the injectivity and surjectivity of the map $H$ are left to the next two sections.

Since the spaces $\mathcal{D}_{P}$ and $\prod_{i=1}^{p} \mathcal{T}_{I_{i}}$ have the same dimension, by using the Brouwer theorem on invariance of domain, we conclude that $H: \mathcal{D}_{P} \rightarrow \prod_{i=1}^{p} \mathcal{T}_{I_{i}}$ is a homeomorphism. The proof of the main theorem is complete.

\section{Injectivity and surJectivity of the map $H$. Part 1}

This section and the next section are devoted to the proofs of the injectivity and surjectivity of $H: \mathcal{D}_{P} \rightarrow \prod_{i=1}^{p} \mathcal{T}_{I_{i}}$. This section gives the proofs of the injectivity and surjectivity of $H$ in case there is at least one face in $\left\{\mathcal{F}_{1}, \mathcal{F}_{2}, \cdots, \mathcal{F}_{p}\right\}$ with exactly three vertices. Since the contact graph $G$ is connected, all faces in $\left\{\mathcal{F}_{i}\right\}_{1 \leq i \leq p}$ are simply connected.

3.1. Some preliminary results. To complete the injectivity and surjectivity of the map $H$, the following two lemmas are needed. They characterize configurations in $\mathbb{C}$ consisting of three circles. We refer to [12, 19] for their complete proofs.

Lemma 3.1. For any three angles $\theta_{1}, \theta_{2}, \theta_{3} \in[0, \pi / 2]$ and any three numbers $r_{1}, r_{2}, r_{3}>0$, there is a configuration of three circles in $\mathbb{C}$, unique up to Euclidean isometries, having radii $\left\{r_{i}\right\}$ and intersecting in angles $\left\{\theta_{i}\right\}$.

Lemma 3.2. Let $c_{1}, c_{2}$ and $c_{3}$ be circles in $\mathbb{C}$ with radii of $r_{1}, r_{2}$ and $r_{3}$. Also we suppose that $c_{1}, c_{2}$ and $c_{3}$ meet pairwise in non-obtuse angles. If $c_{2}$ and $c_{3}$ are held constant, but $c_{1}$ is varied in such a way that the angles of intersection are constant but the radius $r_{1}$ decreases, then

$$
\frac{\partial \alpha_{1}}{\partial r_{1}}<0, \frac{\partial \alpha_{2}}{\partial r_{1}}>0, \frac{\partial \alpha_{3}}{\partial r_{1}}>0,
$$

where $\alpha_{i}, i=1,2,3$, are the corresponding angles of the triangle of centers.

For any interstice $I \in\left\{I_{1}, I_{2}, \cdots, I_{p}\right\}$ with $k(>3)$ circular $\operatorname{arcs}\left\{\gamma_{j}\right\}_{1 \leq j \leq k}$ on its boundary, let $\left\{P\left(v_{1}\right), P\left(v_{2}\right), \cdots, P\left(v_{k}\right)\right\}$ be the corresponding circles in $P$ adjacent to $I$ such that $\gamma_{j}=\partial I \cap P\left(v_{j}\right), 1 \leq j \leq k$. Denote by $\theta_{j} \equiv \Theta\left(\left[v_{j}, v_{j+1}\right]\right)$ the dihedral angle between the intersecting circles $P\left(v_{j}\right)$ and $P\left(v_{j+1}\right)$, where $v_{k+1} \equiv v_{1}$. With this notation, we have the following.

Lemma 3.3. Given a complex structure $[\tau: I \rightarrow \hat{\mathbb{C}}] \in \mathcal{T}_{I}$ and $\left\{r_{j}>0\right\}_{1 \leq j \leq k}$, there exists an immersion $h_{\tau}: I \rightarrow \mathbb{C}$ such that $h_{\tau}=\Phi_{\tau} \circ \tau$ for some analytic immersion $\Phi_{\tau}: \tau(I) \rightarrow \mathbb{C}$. In addition, $h_{\tau}\left(\gamma_{j}\right)$ is a circular arc on a circle $c_{j}^{\tau}$ 
with radius $r_{j}$ and the intersecting angle between the adjacent circles $c_{j}^{\tau}, c_{j+1}^{\tau}$ is $\theta_{j}$, where $1 \leq j \leq k$.

The map $h_{\tau}: I \rightarrow \mathbb{C}$ is uniquely determined up to post-composition with Euclidean isometries.

Remark 3.4. Although the circles $\left\{P\left(v_{j}\right)\right\}$ forming the interstice $I$ are not necessarily distinct, the associated circles $c_{j}^{\tau}$ in the statement are distinct (although in the ultimate immersions, they may necessarily coincide).

Denote $F^{\tau} \subset \mathbb{C}$ to be the immersed $k$-polygon by connecting the centers of the adjacent circles $c_{j}^{\tau}, c_{j+1}^{\tau}$ with straight arcs. It follows from Lemma 3.3 that all angles of the polygon $F^{\tau}$, denoted by $\alpha_{j}^{\tau}$, are well defined, $1 \leq j \leq k$. Let $r_{j}, \tilde{r}_{j}, 1 \leq j \leq k$, be two sets of positive numbers. By using the same complex structure $[\tau: I \rightarrow \hat{\mathbb{C}}] \in \mathcal{T}_{I}$ and the same dihedral angles $\theta_{j} \in[0, \pi / 2], 1 \leq j \leq k$, we obtain two immersed $k$-polygons $F^{\tau}, \tilde{F}^{\tau} \subset \mathbb{C}$. Then we have the following.

Lemma 3.5. Suppose there is a proper subset $\emptyset \neq\left\{j_{1}, j_{2}, \cdots, j_{\mu}\right\} \varsubsetneqq\{1,2, \cdots, k\}$ such that $\tilde{r}_{j_{\lambda}}<r_{j_{\lambda}}, 1 \leq \lambda \leq \mu$, and $\tilde{r}_{j} \geq r_{j}, j \in\{1,2, \cdots, k\} \backslash\left\{j_{1}, j_{2}, \cdots, j_{\mu}\right\}$. Then the angles of the polygons $F^{\tau}$, $\tilde{F}^{\tau}$ satisfy

$$
\sum_{\lambda=1}^{\mu} \tilde{\alpha}_{j_{\lambda}}^{\tau}>\sum_{\lambda=1}^{\mu} \alpha_{j_{\lambda}}^{\tau}
$$

The proofs of Lemmas 3.3 and 3.5 are deferred to Section 3.5

3.2. Sketch of the proof of Theorem 1.3. By our assumption, there is a triangle in $\left\{\mathcal{F}_{1}, \mathcal{F}_{2}, \cdots, \mathcal{F}_{p}\right\}$, say $\mathcal{F}_{p}$, whose boundary edges $\left\{e_{1}, e_{2}, e_{3}\right\}$ satisfy $\sum_{j=1}^{3} \Theta\left(e_{j}\right)<\pi$. It will be convenient to label the vertices in $V$ by $\left(v_{1}, v_{2}, v_{3}, \cdots\right.$, $\left.v_{|V|}\right)$, where $v_{|V|-2}, v_{|V|-1}, v_{|V|}$ are the vertices of the face $\mathcal{F}_{p}$.

Let $r=\left(r_{1}, r_{2}, \cdots, r_{|V|}\right)$ be a vector with $|V|$ positive numbers. With the given data $(G, \Theta)$ and any complex structures $\left\{\tau_{i} \in \mathcal{T}_{I_{i}}\right\}_{1 \leq i \leq p}$, then the vector $r$ determines a polygonal structure on the topological 2-sphere as follows:

For each face $\mathcal{F}_{i}$ with three vertices, say $\left\{v_{1}, v_{2}, v_{3}\right\}$, we associate the triangle determined by the centers of three mutually intersecting circles with radii $r_{1}, r_{2}$ and $r_{3}$ and with intersecting angles $\left\{\Theta\left(\left[v_{1}, v_{2}\right]\right)\right\},\left\{\Theta\left(\left[v_{2}, v_{3}\right]\right)\right\}$ and $\left\{\Theta\left(\left[v_{3}, v_{1}\right]\right)\right\}$; please see Lemma 3.1 .

For any $\mathcal{F}_{i} \in\left\{\mathcal{F}_{1}, \mathcal{F}_{2}, \cdots, \mathcal{F}_{p}\right\}$ with $k(>3)$ vertices, we denote its vertices by $\left\{v_{i_{1}}, v_{i_{2}}, \cdots, v_{i_{k}}\right\}$. With the positive numbers $\left\{r_{i_{j}}>0\right\}_{1 \leq j \leq k}$ and the complex structure $\tau_{i} \in \mathcal{T}_{I_{i}}$, Lemma 3.3 shows that there exists a unique Euclidean k-polygon $F_{i}^{\tau}$ determined by the centers of $k$ circles with radii $\left\{r_{i_{j}}>0\right\}$ and dihedral angles $\left\{\Theta\left(\left[v_{i_{j}}, v_{i_{j+1}}\right]\right)\right\}$, where $v_{i_{k+1}} \equiv v_{i_{1}}$.

Transfer the Euclidean metrics on these Euclidean polygons to the associated faces of $\left\{\mathcal{F}_{1}, \mathcal{F}_{2}, \cdots, \mathcal{F}_{p}\right\}$. This metric is well defined on any edge which is common to two faces (maybe the same). In this way the topological sphere becomes a locally Euclidean space with cone type singularities at the vertices. Denote this metric space by $\mathcal{M}_{r}$. Using the metric of $\mathcal{M}_{r}$, for $1 \leq l \leq|V|$, the apex curvature $k_{r}\left(v_{l}\right)$ at the vertex $v_{l}$ is defined to be $k_{r}\left(v_{l}\right) \equiv 2 \pi-\sigma\left(v_{l}\right)$, where $\sigma\left(v_{l}\right)$ is the sum of the angles at $v_{l}$ of all faces containing $v_{l}$. Therefore

$$
\sum_{l=1}^{|V|} k_{r}\left(v_{l}\right)=\sum_{l=1}^{|V|}\left(2 \pi-\sigma\left(v_{l}\right)\right)=2 \pi \cdot|V|-\sum_{i=1}^{p}\left(\left|E_{i}\right|-2\right) \cdot \pi,
$$


where $E_{i}$ is the edges set of $\mathcal{F}_{i}$. From the Euler formula $|V|-|E|+|F|=2$, it follows that

$$
\sum_{l=1}^{|V|} k_{r}\left(v_{l}\right)=2 \pi \cdot|V|+2 \pi \cdot|F|-2 \pi \cdot|E|=4 \pi .
$$

For $\lambda>0$, the two metric spaces $\mathcal{M}_{r}, \mathcal{M}_{\lambda \cdot r}$ are similar in the sense that the corresponding angles are equal. Therefore $k_{r}\left(v_{l}\right)=k_{\lambda \cdot r}\left(v_{l}\right)$. It turns out to be advantageous to normalize the map

$$
r \rightarrow f(r)=\left(k_{r}\left(v_{1}\right), k_{r}\left(v_{2}\right), \cdots, k_{r}\left(v_{|V|}\right)\right)
$$

by restricting its domain to the simplex

$$
X \equiv\left\{\left(r_{1}, r_{2}, \cdots, r_{|V|}\right) \in \mathbb{R}_{+}^{|V|}: r_{1}+r_{2}+\cdots+r_{|V|}=1\right\} .
$$

It follows from (11) that the range of $f$ can be taken as the hyperplane

$$
Y \equiv\left\{\left(y_{1}, y_{2}, \cdots, y_{|V|}\right) \in \mathbb{R}^{|V|}: y_{1}+y_{2}+\cdots+y_{|V|}=4 \pi\right\} .
$$

To prove the uniqueness and existence assertions of Theorem 1.3 it is sufficient to verify that the map $f: X \rightarrow Y$ is injective and the point

$$
p_{0}=(0,0, \cdots, 0,4 \pi / 3,4 \pi / 3,4 \pi / 3) \in f(X) .
$$

Assume the injectivity of $f$ for the moment, and assume that $f\left(r_{0}\right)=p_{0}$ for some

$$
r_{0}=\left(r_{01}, r_{02}, \cdots, r_{0|V|}\right) \in \mathbb{R}_{+}^{|V|} .
$$

Then the local Euclidean space $\mathcal{M}_{r_{0}}$ is homeomorphic to $\hat{\mathbb{C}}$. Recall that $\left\{e_{1}, e_{2}, e_{3}\right\}$ are the edges of the face $\mathcal{F}_{p}$ with three edges. The loop in $G$ formed by $e_{1}, e_{2}, e_{3}$ divides $\hat{\mathbb{C}}$ into two simply connected regions $\Delta_{1}$ and $\Delta_{2} \equiv \hat{\mathbb{C}} \backslash \mathcal{F}_{p}$. Then there is a local homeomorphism $\phi: \Delta_{2} \rightarrow \mathbb{C}$ (please compare to Lemma 3.8). The image $\phi\left(\Delta_{2}\right)$ is an immersed polygon in $\mathbb{C}$. Note that a local embedding of a topological disk into the complex plane which is an actual embedding on the boundary must be a global embedding. We conclude that the isometric immersions $\phi: \Delta_{2} \rightarrow \mathbb{C}$ is a global embedding.

If we center a circle with radius $r_{0 l}$ at the point $\phi\left(v_{l}\right), 1 \leq l \leq|V|$, from the definition of $\mathcal{M}_{r_{0}}$ it follows that we have an immersed circle pattern in $\mathbb{C}$ with contact graph $G$. Denote by $A_{1}, A_{2}, A_{3}$ the vertex of the polyhedron $\phi\left(\Delta_{2}\right)$. It will be useful when we discuss uniqueness to observe that the triangle $A_{1} A_{2} A_{3}$ is necessarily equilateral. If we weld another copy of triangle $A_{1} A_{2} A_{3}$ along the corresponding edges, then we have an isometric image of all of $\mathcal{M}_{r_{0}}$. The curvature at the vertex $A_{i}$ is $2 \pi$ minus the sum of all angles in the isometric image with the vertex $A_{i}$. By the definition of $\mathcal{M}_{r_{0}}$, the curvature at the vertex $A_{i}, 1 \leq i \leq 3$, must turn out to be $4 \pi / 3$. Thus $4 \pi / 3=2 \pi-2 \cdot m\left(A_{i}\right)$, where $m\left(A_{i}\right)$ is the angle measure of the angle $A_{i}$ in the triangle $A_{1} A_{2} A_{3}$. We conclude that $m\left(A_{i}\right)=\pi / 3, i=1,2,3$. Thus $A_{1} A_{2} A_{3}$ is an equilateral triangle. Stereographic projection transforms this circle pattern to a circle pattern in $\hat{\mathbb{C}}$ with the same property.

The uniqueness assertion of Theorem 1.3 follows from the injectivity of $f: X \rightarrow$ $Y$. Therefore the proof will be complete once we establish the injectivity of $f$ : $X \rightarrow Y$ and claim (5). 
3.3. Proof of the injectivity of $H: \mathcal{D}_{P} \rightarrow \prod_{i=1}^{p} \mathcal{T}_{I_{i}}$. We will show that $f: X \rightarrow$ $Y$ is injective in this subsection.

Let $\tilde{r}=\left(\tilde{r}_{1}, \tilde{r}_{2}, \cdots, \tilde{r}_{|V|}\right)$ and $r=\left(r_{1}, r_{2}, \cdots, r_{|V|}\right)$ be two distinct points in $X$. Let $V_{0} \subset V$ denote the set of vertices $v_{l}$ for which $\tilde{r}_{l}<r_{l}$. Note that the definition of $X$ in (3) implies that $\emptyset \neq V_{0} \varsubsetneqq V$.

Let $\left\{F_{1}, F_{2}, \cdots, F_{p}\right\}$ denote the faces in $\mathcal{M}_{r}$. Denote by $Q_{0} \subset\left\{F_{i}\right\}_{1 \leq i \leq p}$ the subset consisting of all the faces such that there is at least one vertex in $V_{0}$. Write

$$
Q_{0}=Q_{N} \cup Q_{A},
$$

where $F \in Q_{N}$ if and only if a proper subset of its vertices set is in $V_{0}$, and $F \in Q_{A}$ if and only if all of its vertices are in $V_{0}$. We then have

$$
\sum_{v \in V_{0}} k_{r}(v)=\sum_{v \in V_{0}}\left(2 \pi-\sigma_{r}(v)\right)=2 \pi \cdot\left|V_{0}\right|-\sum_{F \in Q_{N}} \sum_{j} \alpha_{j}^{r}-\sum_{F \in Q_{A}} \sum_{j} \beta_{j}^{r},
$$

where $\left\{\alpha_{j}^{r}\right\}$ is the angle at the vertices of $F \in Q_{N}$ included in $V_{0}$, and $\left\{\beta_{j}^{r}\right\}$ is the angle set of the face $F \in Q_{A}$.

For each face $F \in Q_{N}$, Lemma 3.2 (if it has three sides) or Lemma 3.5 (if it has $k(>3)$ sides) implies that if $r$ is replaced by $\tilde{r}$, then

$$
\sum_{j} \alpha_{j}^{r}<\sum_{j} \alpha_{j}^{\tilde{r}}
$$

Also, if a face $F \in Q_{A}$ has $k$ sides, then

$$
\sum_{j} \beta_{j}^{r}=\sum_{j} \beta_{j}^{\tilde{r}}=(k-2) \cdot \pi .
$$

Note that $\emptyset \neq V_{0} \varsubsetneqq V$ and not all faces of $\mathcal{M}_{r}$ are in $Q_{A}$. The combination of (8) and (9) gives

$$
\sum_{v \in V_{0}} k_{r}(v)>\sum_{v \in V_{0}} k_{\tilde{r}}(v),
$$

which implies that $f: X \rightarrow Y$ is injective.

3.4. Proof of the surjectivity of $H: \mathcal{D}_{P} \rightarrow \prod_{i=1}^{p} \mathcal{T}_{I_{i}}$. For any boundary point $s=\left(s_{1}, s_{2}, \cdots, s_{|V|}\right) \in \partial X$, we now examine the behavior of the function $f(r)$ as $r \rightarrow s \in \partial X$. Then there is at least one $s_{l}=0$, where $1 \leq l \leq|V|$. Denote $\mathcal{L}(s) \equiv\left\{1 \leq l \leq|V|: s_{l}=0\right\}$. Therefore $\emptyset \neq \mathcal{L}(s) \varsubsetneqq\{1,2, \cdots,|V|\}$. For simplicity of notation, we sometimes use $\mathcal{L}$ instead of $\mathcal{L}(s)$. Denote

$$
V_{1}^{\mathcal{L}} \equiv\left\{v_{l} \in V: s_{l}=0\right\} .
$$

Then $\emptyset \neq V_{1}^{\mathcal{L}} \varsubsetneqq V$. Recall that $\left\{F_{1}, F_{2}, \cdots, F_{p}\right\}$ are faces of the metric space $\mathcal{M}_{r}$. Denote by

$$
Q_{1} \subset\left\{F_{i}\right\}_{1 \leq i \leq p}
$$

the subset consisting of all the faces such that there is at least one vertex in $V_{1}^{\mathcal{L}}$.

For any face $F \in Q_{1}$ with three vertices, we assume that its vertices are $v_{1}, v_{2}, v_{3}$ $\in V$. As before, let $\alpha_{1}^{r}, \alpha_{2}^{r}, \alpha_{3}^{r}$ be the angles of $F$ at the center of the circles $P\left(v_{1}\right), P\left(v_{2}\right), P\left(v_{3}\right)$. Then as $r \rightarrow s \in \partial X$, we have three cases to distinguish: when only one radius approaches to 0 , say $\rho\left(P\left(v_{1}\right)\right) \rightarrow 0$, then

$$
\alpha_{1}^{r} \rightarrow \pi-\Theta\left(\left[v_{2}, v_{3}\right]\right) \text {. }
$$


When two radii of circles shrink to zero, say $P\left(v_{1}\right), P\left(v_{2}\right)$, then

$$
\alpha_{1}^{r}+\alpha_{2}^{r} \rightarrow \pi \text {. }
$$

When all the radii $\rho\left(P\left(v_{1}\right)\right), \rho\left(P\left(v_{2}\right)\right), \rho\left(P\left(v_{3}\right)\right) \rightarrow 0^{+}$, obviously

$$
\alpha_{1}^{r}+\alpha_{2}^{r}+\alpha_{3}^{r} \equiv \pi \text {. }
$$

For any face $F \in Q_{1}$ with $k(>3)$ sides, without loss of generality we assume that its vertices are

$$
v_{1}, v_{2}, \cdots, v_{k}, v_{k+1} \equiv v_{1}, v_{k+2} \equiv v_{2}, \cdots .
$$

Denote $V_{1}^{F} \equiv V_{1}^{\mathcal{L}} \cap\left\{v_{1}, v_{2}, \cdots, v_{k}\right\}$. If $\left|V_{1}^{F}\right|=k$ or $k-1$, then we can easily obtain

$$
\lim _{r \rightarrow s} \sum_{v_{j} \in V_{1}^{F}} \alpha_{j}^{r} \rightarrow(k-2) \cdot \pi \text {. }
$$

When $\left|V_{1}^{F}\right|=k-2$, if $v_{j_{1}}, v_{j_{2}} \in\left\{v_{1}, v_{2}, \cdots, v_{k}\right\} \backslash V_{1}^{F}$ are a pair of adjacent vertices in the boundary of $F$, then

$$
\sum_{v_{j} \in V_{1}^{F}} \alpha_{i}^{r} \rightarrow(k-2) \cdot \pi-\Theta\left(\left[v_{j_{1}}, v_{j_{2}}\right]\right) .
$$

If $v_{j_{1}}, v_{j_{2}}$ are not a pair of adjacent vertices in the boundary of $F$, then

$$
\sum_{v_{j} \in V_{1}^{F}} \alpha_{i}^{r} \rightarrow(k-2) \cdot \pi .
$$

As $r \rightarrow s \in \partial X$, observing $F^{r} \in Q_{1}$ and all interstices $I_{r}$ have the same given complex structure for all $r$, then we have the Euclidean diameters,

$$
\operatorname{diam}\left(I_{r}\right) \rightarrow 0 \text {. }
$$

If there are $\mu(\geq 3)$ boundary circles of $I_{r}$, say $\left\{v_{j_{1}}, v_{j_{2}}, \cdots v_{j_{\mu}}\right\}$, whose radii do not approach to $0^{+}$, then the $\mu$ limiting circles

$$
\left\{P\left(v_{j_{1}}\right)^{s}, P\left(v_{j_{2}}\right)^{s}, \cdots, P\left(v_{j_{\mu}}\right)^{s}\right\}
$$

will meet in a single point in the limiting case. Mapping this point to $\infty$ by a Möbius transformation, we conclude that their dihedral angles satisfy

$$
\sum_{\lambda=1}^{\mu} \Theta\left(P\left(v_{j_{\lambda}}\right)^{s}, P\left(v_{j_{\lambda+1}}\right)^{s}\right)=(\mu-2) \cdot \pi .
$$

Of course, we have

$$
\Theta\left(P\left(v_{j_{\lambda}}\right)^{s}, P\left(v_{j_{\lambda+1}}\right)^{s}\right)=\Theta\left(\left[v_{j_{\lambda}}, v_{j_{\lambda+1}}\right]\right)
$$

when $j_{\lambda+1}-j_{\lambda}=1$. From Lemma 3.3 and Remark 3.4, it follows that the dihedral angles $\Theta\left(P\left(v_{j_{\lambda}}\right)^{s}, P\left(v_{j_{\lambda+1}}\right)^{s}\right)$ may be $>\pi / 2$ if $j_{\lambda+1}-j_{\lambda}>1$.

Let $\left\{P\left(v_{j_{\lambda}}\right), P\left(v_{j_{\lambda}+1}\right), P\left(v_{j_{\lambda}+2}\right), \cdots, P\left(v_{j_{\lambda+1}}\right)\right\}$ be any boundary circles chain with $j_{\lambda+1}-j_{\lambda}>1$, where $1 \leq \lambda \leq \mu$. If $j_{\lambda}<j<j_{\lambda+1}$, then their radii

$$
\rho\left(P\left(v_{j}\right)^{r}\right) \rightarrow 0
$$

as $r \rightarrow s \in \partial X$. As before, let $\alpha_{j}^{r}, j_{\lambda}<j<j_{\lambda+1}$, denote the angles of $F^{r}$ at the center of the circles $P\left(v_{j}\right)^{r}$. By a simple computation, as $r \rightarrow s \in \partial X$,

$$
\sum_{j_{\lambda}<j<j_{\lambda+1}} \alpha_{j}^{r} \rightarrow\left(j_{\lambda+1}-j_{\lambda}-1\right) \cdot \pi+\Theta\left(P\left(v_{j_{\lambda}}\right)^{s}, P\left(v_{j_{\lambda+1}}\right)^{s}\right) ;
$$


please see Figure 4. From (21) it follows that

$$
\lim _{r \rightarrow s} \sum_{v_{j} \in V_{1}^{F}} \alpha_{j}^{r} \rightarrow(k-\mu) \cdot \pi+\sum_{j_{\lambda+1}-j_{\lambda}>1} \Theta\left(P\left(v_{j_{\lambda}}\right)^{s}, P\left(v_{j_{\lambda+1}}\right)^{s}\right) .
$$

In view of (19), (20) and (22), we have

$$
\begin{gathered}
\lim _{r \rightarrow s} \sum_{v_{j} \in V_{1}^{F}} \alpha_{j}^{r} \rightarrow(k-2) \cdot \pi-\sum_{j_{\lambda+1}-j_{\lambda}=1} \Theta\left(P\left(v_{j_{\lambda}}\right)^{s}, P\left(v_{j_{\lambda+1}}\right)^{s}\right) \\
=(k-2) \cdot \pi-\sum_{j_{\lambda+1}-j_{\lambda}=1} \Theta\left(\left[v_{j_{\lambda}}, v_{j_{\lambda+1}}\right]\right) .
\end{gathered}
$$

Obviously we can view (13) $-(15)$ and (16) $-(18)$ as special cases of (23).

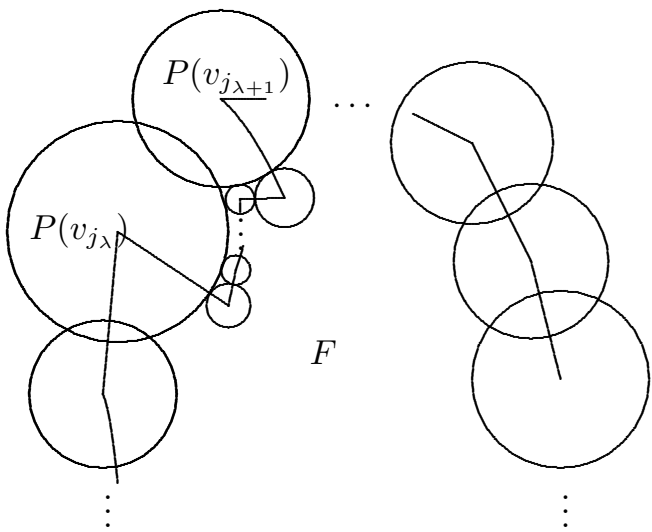

Figure 4

Recall that $Q_{1} \subset\left\{F_{i}\right\}_{1<i<p}$ is the subset consisting of all the faces of $M_{r}$ such that there is at least one vertex in $V_{1}^{\mathcal{L}}$. Denote by $\mathcal{Q}_{1}(\mathcal{L}) \subset\left\{\mathcal{F}_{i}\right\}$ the surfaces associated with $Q_{1}$. By summing up (23) over all faces of the metric space $M_{r}$,

$$
\lim _{r \rightarrow s \in \partial X} \sum_{v \in V_{1}^{\mathcal{L}}} k_{r}(v)=2 \pi|\mathcal{L}|-\pi \cdot \sum_{\mathcal{F} \in \mathcal{Q}_{1}(\mathcal{L})}\left(k_{\mathcal{F}}-2\right)+\sum_{\mathcal{F} \in \mathcal{Q}_{1}(\mathcal{L})} \sum_{e_{\mathcal{F}} \in\left\{e_{\mathcal{F}}\right\}} \Theta\left(e_{\mathcal{F}}\right),
$$

where $k_{\mathcal{F}}$ is the number of edges of the face $\mathcal{F}$, and $\left\{e_{\mathcal{F}}\right\}$ consists of all edges of $\mathcal{F}$ whose end points are both in $V \backslash V_{1}^{\mathcal{L}}$.

For any non-empty proper subset $\mathcal{L} \varsubsetneqq\{1 \leq l \leq|V|\}$, denote by $Y(\mathcal{L}) \subset Y$ the half space

$$
\left\{y: \sum_{l \in \mathcal{L}} y_{l}>2 \pi|\mathcal{L}|-\pi \cdot \sum_{\mathcal{F} \in \mathcal{Q}_{1}(\mathcal{L})}\left(k_{\mathcal{F}}-2\right)+\sum_{\mathcal{F} \in \mathcal{Q}_{1}(\mathcal{L})} \sum_{e_{\mathcal{F}} \in\left\{e_{\mathcal{F}}\right\}} \Theta\left(e_{\mathcal{F}}\right)\right\},
$$

where $y=\left(y_{1}, y_{2}, \cdots, y_{|V|}\right)$. Then we have the following lemma.

Lemma 3.6. The image of the map $f: X \rightarrow Y$ is

$$
f(X)=Y \cap \bigcap_{\mathcal{L}} Y(\mathcal{L}),
$$

where $\mathcal{L}$ varies over all non-empty proper subsets of $\{1,2, \cdots,|V|\}$. 
Proof. Let $Y_{0} \equiv Y \cap \bigcap_{\mathcal{L}} Y(\mathcal{L})$. From (10) and (24) it follows that the image $f(X)$ lies in the convex polyhedron $Y_{0}$. From (24), we have seen that the accumulation points of $f(r)$ as $r \rightarrow \partial X$ lie in the hyperplanes

$$
\sum_{l \in \mathcal{L}} y_{l}=2 \pi|\mathcal{L}|-\pi \cdot \sum_{\mathcal{F} \in \mathcal{Q}_{1}(\mathcal{L})}\left(k_{\mathcal{F}}-2\right)+\sum_{\mathcal{F} \in \mathcal{Q}_{1}(\mathcal{L})} \sum_{e_{\mathcal{F}} \in\left\{e_{\mathcal{F}}\right\}} \Theta\left(e_{\mathcal{F}}\right),
$$

which form the boundary of $Y_{0}$.

Since $f: X \rightarrow Y_{0}$ is a continuous, one-to-one mapping, by invariance of the domain, the map $f: X \rightarrow Y_{0}$ is a homeomorphism.

Therefore $f(X)=Y \cap \bigcap_{\mathcal{L}} Y(\mathcal{L})$.

The rest of this subsection is devoted to proving the following.

Theorem 3.7. The point $p_{0}=(0,0, \cdots, 0,4 \pi / 3,4 \pi / 3,4 \pi / 3)$ is in the image $f(X)$.

It implies the surjectivity of the map $H: \mathcal{D}_{P} \rightarrow \prod_{i=1}^{p} \mathcal{T}_{I_{i}}$.

Proof. Lemma 3.6 implies that we only need to check that $p_{0} \in Y(\mathcal{L})$ for any nonempty proper subset $\mathcal{L}$ of $\{1,2, \cdots,|V|\}$. If $\mathcal{L} \neq \emptyset$, we denote by $V_{1}^{\mathcal{L}} \subset V$ the subset which is defined in (11). To simplify the following computation, we extend the contact graph $G$ to a triangulation $\mathcal{G} \equiv \mathcal{G}(\mathcal{L})$ of $\hat{\mathbb{C}}$ by adding some new edges as follows.

(1) If a face $\mathcal{F} \in\left\{\mathcal{F}_{i}\right\}$ has three vertices, there is nothing to do.

(2) If $\mathcal{F}$ has at least four vertices and it has no vertices in $V_{1}^{\mathcal{L}}$, then a triangulation of $\mathcal{F}$ is given by adding $k-3$ edges in $\mathcal{F}$ (e.g. $\left.\left\{\left[v_{1}, v_{j}\right]\right\}_{3 \leq j \leq k-1}\right)$ to connect the vertices of $\mathcal{F}$.

(3) If $\mathcal{F} \in\left\{\mathcal{F}_{i}\right\}$ has at least $k>3$ vertices and it has at least one vertex in $V_{1}^{\mathcal{L}}$, say $v_{1} \in V_{1}^{\mathcal{L}}$, then we give a triangulation of $\mathcal{F}$ by adding $k-3$ edges in $\mathcal{F}$ (e.g. $\left.\left\{\left[v_{1}, v_{j}\right]\right\}_{3 \leq j \leq k-1}\right)$ to connect the vertices of $\mathcal{F}$.

Let $V_{\mathcal{G}}\left(\right.$ resp. $\left.E_{\mathcal{G}}, F_{\mathcal{G}}\right)$ denote the 0-complex (resp. 1-complex, 2-complex) of $\mathcal{G}$. Obviously $V_{\mathcal{G}} \equiv V$. Define a new function $\theta$ on the edges set by letting $\theta(e)=\Theta(e)$ if $e$ is an edge of $G$, and $\theta(e)=0$ if $e$ is a new edge of $\mathcal{G} \backslash G$. Note the triangulation $\mathcal{G} \equiv \mathcal{G}(\mathcal{L})$ may not be a contact graph because it may have double edges. Also note that $\mathcal{G}$ and $\theta$ are not geometric and they are only devices for computing angle sums. By using $\mathcal{G}$ and $\theta$, we can greatly simplify the computation in this proof.

For any face $\mathcal{F} \in \mathcal{Q}_{1}(\mathcal{L})$, using the triangulation $\mathcal{G}$, the term $\left(k_{\mathcal{F}}-2\right)$ on the right side of the claim (25) can be rewritten as

$$
k_{\mathcal{F}}-2=\text { no. of } 2 \text {-complexes in } F_{\mathcal{G}} \text { which are subfaces of } \mathcal{F} \text {. }
$$

Also, when $v_{i_{j}}, v_{i_{j+1}} \notin V_{1}^{\mathcal{L}}$ and $i_{j+1}-i_{j}=1$, the 2-complex $\left\{v_{1}, v_{i_{j}}, v_{i_{j+1}}\right\} \in F_{\mathcal{G}}$. Also,

$$
\Theta\left(\left[v_{i_{j}}, v_{i_{j+1}}\right]\right)=\theta(e),
$$

where $e \equiv\left[v_{i_{j}}, v_{i_{j+1}}\right] \in E_{\mathcal{G}}$. The 2-complex $\left\{v_{1}, v_{i_{j}}, v_{i_{j+1}}\right\}$ has only one vertex $v_{1}$ in the vertices subset $V_{1}^{\mathcal{L}}$. Denote by $F_{\mathcal{G}}^{\mathcal{L}} \subset F_{\mathcal{G}}$ the set of 2-complexes with only one 
vertex in $V_{1}^{\mathcal{L}}$. By using $\mathcal{G}$ and $\theta$, we can rewrite (25) as

$$
\begin{aligned}
Y(\mathcal{L})=\left\{\sum_{l \in \mathcal{L}} y_{l}>2 \pi|\mathcal{L}|-\pi \cdot(\text { no. of 2-complexes }\right. \\
\left.\left.\quad \text { in } F_{\mathcal{G}} \text { with a vertex in } V_{1}^{\mathcal{L}}\right)+\sum_{\Delta \in F_{\mathcal{G}}^{\mathcal{L}}} \theta\left(e_{\Delta}\right)\right\},
\end{aligned}
$$

where $e_{\Delta} \in E_{\mathcal{G}}$ is the unique edge opposite the vertex of $\Delta$ lying in $V_{1}^{\mathcal{L}}$; see e.g. [12, 19. In view of the construction of $\mathcal{G}$, all new edges of $\mathcal{G}$ are not in the set $\left\{e_{\Delta}\right\}$. Therefore $\theta\left(e_{\Delta}\right)=\Theta\left(e_{\Delta}\right)$.

The following process is analogous to [12. For the sake of completeness we give it here. To prove that $p_{0}=(0,0, \cdots, 0,4 \pi / 3,4 \pi / 3,4 \pi / 3) \in Y(\mathcal{L})$, by using (28), we only need to check that, for any $\emptyset \neq \mathcal{L} \varsubsetneqq\{1 \leq l \leq|V|\}$,

$\sum_{l \in \mathcal{L}} p_{l}>2 \pi|\mathcal{L}|-\pi \cdot\left(\right.$ no. of 2 -complexes in $F_{\mathcal{G}}$ with a vertex in $\left.V_{1}^{\mathcal{L}}\right)+\sum_{\Delta \in F_{\mathcal{G}}^{\mathcal{L}}} \theta\left(e_{\Delta}\right)$,

where $p_{0}=\left(p_{1}, p_{2}, \cdots, p_{|V|}\right)$.

If $|\mathcal{L}|=|V|-1$, then (29) obviously holds. Indeed, there are no faces in $F_{\mathcal{G}}^{\mathcal{L}}$. Hence

$$
\sum_{\Delta \in F_{\mathcal{G}}^{\mathcal{L}}} \theta\left(e_{\Delta}\right)=0
$$

On the other hand, each face of $F_{\mathcal{G}}$ has a vertex in $V_{1}^{\mathcal{L}}$. Therefore, by using the Euler formula, the right-hand side of (29) is

$$
2 \pi \cdot(|V|-1)-\pi \cdot\left|F_{\mathcal{G}}\right|=2 \pi .
$$

The left side of (29) is $8 \pi / 3$ or $12 \pi / 3$. Therefore $p_{0}$ satisfies the inequality (29).

If $|\mathcal{L}|=|V|-2$, then the left-hand side of (29) is at least $4 \pi / 3$. The Euler formula implies

$2 \pi|\mathcal{L}|-\pi \cdot\left(\right.$ no. of faces in $F_{\mathcal{G}}$ with a vertex in $\left.V_{1}^{\mathcal{L}}\right)=2 \pi \cdot(|V|-2)-\pi \cdot\left|F_{\mathcal{G}}\right|=0$.

We have $\sum_{\Delta \in F_{\mathcal{G}}^{\mathcal{G}}} \theta\left(e_{\Delta}\right) \leq \pi$ since there can be at most two faces in the set $F_{\mathcal{G}}^{\mathcal{L}}$. Thus (29) holds in this case as well.

When $1 \leq|\mathcal{L}| \leq|V|-3$, we shall show that $p_{0}$ satisfies (29) by proving that the right-hand side of (29) is negative in these cases. Let $f_{1}, f_{2}, f_{3}$ denote, respectively, the number of faces in $F_{\mathcal{G}}$ which have exactly $1,2,3$ vertices in $V_{1}^{\mathcal{L}}$. In fact we have $f_{1}=\left|F_{\mathcal{G}}^{\mathcal{L}}\right|$. Then

(30) $2 \pi|\mathcal{L}|-\pi \cdot\left(\right.$ no. of faces in $F_{\mathcal{G}}$ with a vertex in $\left.V_{1}^{\mathcal{L}}\right)=\pi \cdot\left(2|\mathcal{L}|-f_{1}-f_{2}-f_{3}\right)$.

Let $\epsilon_{2}$ denote the number of edges of $\mathcal{G}$ which have both of their boundary vertices in $V_{\mathcal{L}}$. Then the simplicial complex $\mathcal{C}_{\mathcal{L}}$ spanned by the vertices of $V_{1}^{\mathcal{L}}$ has Euler characteristic $\chi_{\mathcal{L}}=|\mathcal{L}|-\epsilon_{2}+f_{3}$. Since

$$
3 f_{3}+f_{2}=2 \epsilon_{2},
$$

we have $2 \chi_{\mathcal{L}}=2|\mathcal{L}|-f_{3}-f_{2}$. Hence (29) can be rewritten as

$$
\sum_{l \in \mathcal{L}} p_{l}>\pi \cdot\left(2 \chi_{\mathcal{L}}-f_{1}\right)+\sum_{\Delta \in F_{\mathcal{G}}^{\mathcal{L}}} \theta\left(e_{\Delta}\right)=2 \pi \cdot \chi_{\mathcal{L}}-\sum_{\Delta \in F_{\mathcal{G}}^{\mathcal{L}}}\left(\pi-\theta\left(e_{\Delta}\right)\right),
$$


where $\pi / 2 \leq \pi-\theta\left(e_{\Delta}\right) \leq \pi$ is taken over all the edges of the triangles $\Delta \in F_{\mathcal{G}}^{\mathcal{L}}$ opposite the angle at the vertex in $V_{\mathcal{L}}$.

To prove that the right-hand side of (31) is negative, we may assume that the complex $\mathcal{C}_{\mathcal{L}}$ is connected (therefore $\chi_{\mathcal{L}} \leq 1$ ). Unless $\mathcal{C}_{\mathcal{L}}$ has Euler characteristic 0 or 1 , both terms on the right-hand side of (31) are non-positive, and the sum is negative. If $\chi_{\mathcal{L}}=0$, then $\left|F_{\mathcal{G}}^{\mathcal{L}}\right| \geq 1$ (see e.g. $\S 8$ in [12]). The negative of the right-hand side of (31) is clear. If $\chi_{\mathcal{L}}=1$, then the connectivity of $\mathcal{C}_{\mathcal{L}}$ is 1 . That is, all vertices of $V \backslash V_{\mathcal{L}}$ are contained in a connected component of the graph $\mathcal{G}$. Then $\left|F_{\mathcal{G}}^{L}\right| \geq 3$ (see e.g. $\S 8$ in [12]).

The right-hand side of (31) will also be negative if $\chi_{\mathcal{L}}=1$ and $\left|F_{\mathcal{G}}^{L}\right| \geq 5$.

The remaining cases $\chi_{\mathcal{L}}=1$ and $\left|F_{\mathcal{G}}^{\mathcal{L}}\right|=3$ or 4 are covered by conditions (i), (ii) and (iii) of Section 1. In case $\left|F_{\mathcal{G}}^{\mathcal{L}}\right|=3$, if at least one of the vertices $v_{|V|-2}, v_{|V|-1}, v_{|V|}$ are in $V_{1}^{\mathcal{L}}$, then the left-hand side of (31) is at least $4 \pi / 3$ and the desired inequality obviously holds, even though the right-hand side may be 0 . When $v_{|V|-2}, v_{|V|-1}, v_{|V|} \notin V_{1}^{\mathcal{L}}$, let $\left\{v_{1}^{*}, \cdots, v_{n}^{*}\right\}$ be the adjacent vertices set of $\mathcal{C}_{\mathcal{L}}$. Since $\left|F_{\mathcal{G}}^{\mathcal{L}}\right|=3$, we have $n=3$. If $\left\{v_{1}^{*}, v_{2}^{*}, v_{3}^{*}\right\}=\left\{v_{|V|-2}, v_{|V|-1}, v_{|V|}\right\}$, then by using (iii) in Section 1 and the assumption at the beginning of Subsection 3.2, we have $\sum_{j=|V|-2}^{|V|} \Theta\left(\left[v_{j}, v_{j+1}\right]\right)<\pi$, which is the desired result. When $\left\{v_{1}^{*}, v_{2}^{*}, v_{3}^{*}\right\} \neq\left\{v_{|V|-2}, v_{|V|-1}, v_{|V|}\right\}$, the loop formed by three edges

$$
\left[v_{1}^{*}, v_{2}^{*}\right],\left[v_{2}^{*}, v_{3}^{*}\right],\left[v_{3}^{*}, v_{1}^{*}\right]
$$

separates the set $V$. If at least one of the edges of (32) is in $\mathcal{G} \backslash G$, observing that $\left\{v_{1}^{*}, v_{2}^{*}, v_{3}^{*}\right\} \subset V \backslash V_{1}^{\mathcal{L}}$ is adjacent to $\mathcal{C}_{\mathcal{L}}$, this contradicts the rule (2) of our construction of $\mathcal{G}$. Therefore, $\left[v_{1}^{*}, v_{2}^{*}\right],\left[v_{2}^{*}, v_{3}^{*}\right],\left[v_{3}^{*}, v_{1}^{*}\right] \in G$. By using $(i)$ of Section 1 , we show that the right-hand side of (31) is negative.

Similarly, by using (ii), we conclude that the right-hand of (31) is negative when $\chi_{\mathcal{L}}=1$ and $\left|F_{\mathcal{G}}^{\mathcal{L}}\right|=4$.

Therefore, the point $p_{0}=(0,0, \cdots, 0,4 \pi / 3,4 \pi / 3,4 \pi / 3) \in f(X)$.

3.5. Proof of Lemmas 3.3 and 3.5. Before giving the proofs of Lemmas 3.3 and 3.5, we give some notation.

Let $G_{*}=\left(V_{*}, E_{*}\right)$ be an embedded graph isomorphic to the skeleton of a triangulation $T$ of a closed topological disk. A vertex $v_{0} \in V_{*}$ is called interior if there is a closed chain of neighboring vertices $v_{1}, v_{2}, \cdots, v_{k}, v_{k+1}=v_{1}$, where either $k \geq 4$ or $k=3$ and each edge $\left[v_{0}, v_{j}\right], 1 \leq j \leq 3$, is irreducible. Otherwise it is called a boundary vertex. If a boundary component of $G_{*}$ has $k(>3)$ vertices, and if conditions $(i)$ and $(i i)$ in Section 1 hold, then a vertex is interior precisely when it lies in the interior of the closed topological disk.

To prove Lemmas 3.3 and 3.5 , the following lemma is needed. It is a straight generalization of Andreev's theorem as interpreted by Thurston in [19].

Lemma 3.8. Suppose that $\Theta_{*}: E_{*} \rightarrow[0, \pi / 2]$ is a dihedral angle function. And suppose that conditions $(i),($ ii) and ( iii) in Section 1 hold. For any boundary vertex $v$ of $G_{*}$, let $\rho(v)>0$ be given. Then there is an immersed circle pattern $Q$ in the complex plane $\mathbb{C}$ realizing the data $\left(G_{*}, \Theta_{*}\right)$, such that $\rho(Q(v))=\rho(v)$ for each boundary vertex $v \in V_{*}$.

Moreover the circle pattern $Q$ is unique up to Euclidean isometries. 
Proof. For completeness, we sketch an elementary proof here. For its detail, we refer to [6, 19 .

Denote by $N$ the cardinality of the interior vertices set $V_{*}^{i n t}$. Let $\mathbb{R}_{+}^{N}$ denote the space of functions $\psi: V_{*}^{\text {int }} \rightarrow \mathbb{R}_{+}=(0,+\infty)$. Similarly, denote by $\mathbb{R}^{N}$ the set of functions $K: V_{*}^{\text {int }} \rightarrow \mathbb{R}$.

Then we can construct a transformation $f: \mathbb{R}_{+}^{N} \rightarrow \mathbb{R}^{N}$ as follows.

For any $\psi \in \mathbb{R}_{+}^{N}$, we define $\kappa: V_{*} \rightarrow \mathbb{R}_{+}$by $\kappa(v)=\psi(v)$ for $v \in V_{*}^{\text {int }}$ and $\kappa(v)=\rho(v)$ for each boundary vertex $v \in V_{*}$. Then $\kappa$ defines a path metric in the complex space $T^{(1)}$. In this metric space we have the curvature

$$
K(v)=2 \pi-\sigma(v), \quad v \in V_{1}^{i n t},
$$

where $\sigma(v)$ is the sum of the angles at $v$ of the 2-simplexes which contain the vertex $v$. Then our transformation $f$ takes $\psi$ to the curvature function $K$. By the same argument as in [19], we deduce that $f$ is one-to-one, and maps $\mathbb{R}_{+}^{N}$ onto some open domain in the space $\mathbb{R}^{N}$ bounded by a finite number of hyperplanes. By using conditions $(i)$ and $(i i)$, we deduce that the curvature $K=0 \in \mathbb{R}^{N}$ is always in the correct side of the hyperplanes. Hence it is in the image of the map $f$. Then the corresponding pre-image $\kappa: V_{*} \rightarrow \mathbb{R}_{+}$will define an immersed circle pattern $Q$ in $\mathbb{C}$, which realizes the data $\left(G_{*}, \Theta_{*}\right)$ and satisfies the required boundary condition.

Clearly, the uniqueness of $Q$ up to Euclidean isometries follows from the uniqueness of the map $\kappa$.

Proof of Lemma 3.3. Now we can prove the existence part of this lemma.

Note that $\left\{P\left(v_{1}\right), P\left(v_{2}\right), \cdots, P\left(v_{k}\right)\right\}$ are circles which share circular arcs $\left\{\gamma_{1}, \gamma_{2}, \cdots, \gamma_{k}\right\}$ with the boundary of the interstice $I$. Also there are $k$ marked vertices on $\partial I$, which are the intersecting points of the circles $P\left(v_{j}\right)$ and $P\left(v_{j+1}\right)$, where $1 \leq j \leq k$.

For the given complex structure $[\tau: I \rightarrow \hat{\mathbb{C}}]$, there are $k$ vertices on $\tau(I)$, denoted by $\left\{a_{1}, a_{2}, \cdots, a_{k}\right\}$. By post-composition with a Mobiüs transformation, we may assume that the region $\tau(I) \subset \hat{\mathbb{C}}$ is a bounded domain in the complex plane $\mathbb{C}$. Lay down a regular hexagonal packing of circles in $\mathbb{C}$, say each of radius $1 / n$. By a small translation, we may assume that each boundary vertex of $\left\{a_{1}, a_{2}, \cdots, a_{k}\right\}$ lies inside a circle. By using the boundary component $\partial \tau(I)$ like a cookie-cutter, we obtain a circle packing $Q_{n}$ which consists of all the circles intersecting the closed region $\tau(I)$. The circles in $Q_{n}$ which meet the boundary $\partial \tau(I)$ will be called boundary circles of $Q_{n}$.

Denote by $K_{n}$ the contact graph of $Q_{n}$. By using the contact graph $K_{n}$, we construct a new graph $\mathcal{K}_{n}$ as follows. For $j=1,2, \cdots, k$, we add the vertex $v_{j}$ (using the same notation) to the graph $K_{n}$, and add edges $\left[v_{j}, v_{j+1}\right], 1 \leq j \leq k$, where $v_{k+1} \equiv v_{1}$. Then we add edges $\left[v_{j}, v\right]$, where $v \in\left\{v \in \partial K_{n}: Q_{n}(v) \cap \tau\left(\gamma_{i}\right) \neq \emptyset\right\}$. Obviously the resulting graph $\mathcal{K}_{n}$ is isomorphic to the 1-skeleton of a triangulation of a closed topological disk.

Let $\mathcal{E}_{n}$ denote the edge set of $\mathcal{K}_{n}$. Define a dihedral angle function $\theta_{n}: \mathcal{E}_{n} \rightarrow$ $[0, \pi / 2]$ by setting $\theta_{n}(e)=\Theta(e)$ if $e=\left[v_{j}, v_{j+1}\right], 1 \leq j \leq k$. Otherwise set $\theta_{n}(e)=0$. Since $k>3$, we can easily check that $\left(\mathcal{K}_{n}, \theta_{n}\right)$ satisfies conditions $(i),(i i)$ and (iii). Lemma 3.8 implies that there exists a unique immersed circle pattern $\mathcal{Q}_{n}$ in $\mathbb{C}$ realizing $\left(\mathcal{K}_{n}, \theta_{n}\right)$. Also its boundary circles have radii $\rho\left(\mathcal{Q}_{n}\left(v_{j}\right)\right)=r_{j}, 1 \leq j \leq k$. 
Let $F_{n}^{\tau}$ be the carriel 2 of the circle packing $Q_{n}$. Then we have a simplicial map $A_{n}: F_{n}^{\tau} \rightarrow \mathbb{C}$ determined by the correspondence of centers of circles in the circle patterns $Q_{n}$ and $\mathcal{Q}_{n}$. By the Ring Lemma in [14, $A_{n}: F_{n}^{\tau} \rightarrow \mathbb{C}$ is a quasiregular function. Letting $n \rightarrow \infty$, the following Proposition 3.9 and the quasicomformality implies that $\left\{A_{n}\right\}$ will converge to an analytic immersion $A_{\tau}: \tau(I) \rightarrow \mathbb{C}$; see e.g. [14. It is clear that the map $h_{\tau} \equiv A_{\tau} \circ \tau: I \rightarrow \mathbb{C}$ has the desired properties.

Proposition 3.9. The analytic map $A_{\tau}: \tau(I) \rightarrow \mathbb{C}$ does not degenerate.

Proof. Observe that $\left\{\mathcal{Q}_{n}\left(v_{1}\right), \mathcal{Q}_{n}\left(v_{2}\right), \cdots, \mathcal{Q}_{n}\left(v_{k}\right)\right\}$ are the boundary circles of $\mathcal{Q}_{n}$, and for all $n \geq 1$ they have radii $\rho\left(\mathcal{Q}_{n}\left(v_{j}\right)\right)=r_{j}, 1 \leq j \leq k$. If the limit $A_{\tau}$ : $\tau(I) \rightarrow \mathbb{C}$ degenerates to a constant $z_{0}$, then the circle patterns $\left\{\mathcal{Q}_{n}\right\}$ will converge to a circle pattern $\mathcal{Q}$ with $\rho\left(\mathcal{Q}\left(v_{j}\right)\right)=r_{j}, 1 \leq j \leq k$ and all circles $\left\{\mathcal{Q}\left(v_{j}\right)\right\}$ meet at $z_{0}$.

By using a Möbius transformation, we map $z_{0}$ to $\infty$. Under this transformation, the new circle pattern $\mathcal{Q}$ (use the same notation) meets at $\infty$. Therefore all circles of $\mathcal{Q}$ are straight lines. Since $k \geq 4, \mathcal{Q}\left(v_{1}\right)$ and $\mathcal{Q}\left(v_{3}\right)$ are a pair of parallel lines in $\mathbb{C}$. Also $\mathcal{Q}\left(v_{2}\right)$ and $\mathcal{Q}\left(v_{4}\right)$ are a pair of parallel lines. Therefore $k=4$ and $\sum_{j=1}^{4} \Theta\left(\left[v_{j}, v_{j+1}\right]\right)=2 \pi$. Hence $\Theta\left(\left[v_{j}, v_{j+1}\right]\right)=\pi / 2,1 \leq j \leq 4$. By condition $(i i)$, this contradicts our assumption that $I$ is an interstice of the fixed circle pattern $P$ with $k(>3)$ vertices.

To show the uniqueness part of Lemma 3.3, we assume, by contradiction, that $h_{\tau}: I \rightarrow \mathbb{C}$ and $\tilde{h}_{\tau}: I \rightarrow \mathbb{C}$ are two immersed maps with the desired properties stated in Lemma 3.3

For simplicity, at first we assume $h_{\tau}, \tilde{h}_{\tau}$ are injective. Then there exists a holomorphic homeomorphism $\phi \equiv \tilde{h}_{\tau} \circ h_{\tau}^{-1}: h_{\tau}(I) \rightarrow \tilde{h}_{\tau}(I)$. Obviously the homeomorphism $\phi$ can be extended to a homeomorphism between the closures of $h_{\tau}(F), \tilde{h}_{\tau}(F)$, denoted by the same notation $\phi$.

Since $h_{\tau}, \tilde{h}_{\tau}$ map the circular arc $\gamma_{j} \subset \partial I$ to circular arcs with the same radius $r_{j}$, where $1 \leq j \leq k$. Then the harmonic function $\log \left|\phi^{\prime}(w)\right|, w \in h_{\tau}(I)$, satisfies the following Dirichlet-Neumann boundary condition

$$
\frac{\partial}{\partial n} \log \left|\phi^{\prime}(w)\right|=\frac{1}{r_{j}}-\frac{\left|\phi^{\prime}(w)\right|}{r_{j}}, \quad w \in h_{\tau}\left(\gamma_{j}\right), \quad 1 \leq j \leq k,
$$

where the operator $\partial / \partial n$ denotes differentiation with respect to the exterior normal on the boundary curve of $h_{\tau}(I)$. For more details, please refer to the Appendix of this paper.

Suppose that the harmonic function $\log \left|\phi^{\prime}(w)\right|$ attains its minimal at a point $w_{0} \in \partial h_{\tau}(I)$. Then $w_{0} \in h_{\tau}\left(\gamma_{j}\right)$ for some $1 \leq j \leq k$. There are three cases to distinguish:

(1) When $w_{0}$ is not an intersecting point of two adjacent boundary circles, we have

$$
0 \geq \frac{\partial}{\partial n} \log \left|\phi^{\prime}\left(w_{0}\right)\right|=\frac{1}{r_{j}}-\frac{\left|\phi^{\prime}\left(w_{0}\right)\right|}{r_{j}},
$$

from which we deduce that $\left|\phi^{\prime}\left(w_{0}\right)\right| \geq 1$.

\footnotetext{
${ }^{2}$ The carrier of a circle packing is by definition the union of all interior of the circles and all interstices of the circle pattern.
} 
(2) If $w_{0}$ is an intersecting of two adjacent circles with the dihedral angle $\neq 0$, then

Hence $\left|\phi^{\prime}\left(w_{0}\right)\right| \geq 1$.

$$
0 \geq \frac{\partial}{\partial n} \log \left|\phi^{\prime}\left(w_{0}\right)\right|=\frac{1}{r_{j}}-\frac{\left|\phi^{\prime}\left(w_{0}\right)\right|}{r_{j}} .
$$

(3) If $w_{0}$ is the tangent point of two adjacent circles, simple computation shows that $\left|\phi^{\prime}\left(w_{0}\right)\right|=1$.

The above three cases imply that $\left|\phi^{\prime}(w)\right| \geq 1, \forall w \in h_{\tau}(I)$.

Similarly, by considering the point where $\log \left|\phi^{\prime}(w)\right|$ attains its maximal value, we deduce that $\left|\phi^{\prime}(w)\right| \leq 1, \forall w \in h_{\tau}(I)$. Therefore, $\left|\phi^{\prime}(w)\right| \equiv 1, \forall w \in h_{\tau}(I)$, which shows $\tilde{h}_{\tau}=g \circ h_{\tau}$ for some Euclidean similarity $g$.

When either of the immersions $h_{\tau}, \tilde{h}_{\tau}$ is not a holomorphic homeomorphism, we use the Riemann region $\mathcal{D}_{\tau} \equiv\left\{\left(z, h_{\tau}(z)\right) \subset \mathbb{C}^{2}: z \in I\right\}$ (resp. $\tilde{\mathcal{D}}_{\tau} \equiv\left\{\left(z, \tilde{h}_{\tau}(z)\right) \subset\right.$ $\left.\mathbb{C}^{2}: z \in I\right\}$ ) rather than the region $h_{\tau}(I)$ (resp. $\tilde{h}_{\tau}(I)$ ); see e.g. $\S 1.4$ in [17] for the definition. In this case the proof is essentially a repetition of the above so it is omitted. Therefore this completes the proof of Lemma 3.3.

Proof of Lemma 3.5. As in the proof of Lemma 3.3. we assume that $h_{\tau}: I \rightarrow$ $h_{\tau}(I), \tilde{h}_{\tau}: I \rightarrow \tilde{h}_{\tau}(I)$ are both global holomorphic homeomorphisms. Then there exists a holomorphic homeomorphism

$$
\phi \equiv \tilde{h}_{\tau} \circ h_{\tau}^{-1}: h_{\tau}(I) \rightarrow \tilde{h}_{\tau}(I) .
$$

Obviously the holomorphic homeomorphism $\phi$ can be extended to a homeomorphism between their closures. Then the harmonic function $\log \left|\phi^{\prime}(w)\right|$ satisfies

$$
\frac{\partial}{\partial n} \log \left|\phi^{\prime}(w)\right|=\frac{1}{r_{j}}-\frac{\left|\phi^{\prime}(w)\right|}{\tilde{r}_{j}}, w \in h_{\tau}\left(\gamma_{j}\right), 1 \leq j \leq k,
$$

where $r_{j}$ (resp. $\tilde{r}_{j}$ ) is the radius of the circle $c_{j}^{\tau}$ (resp. $\tilde{c}_{j}^{\tau}$ ). Let $w_{0}$ be the point where $\left|\phi^{\prime}\left(w_{0}\right)\right|$ attains its maximal value. Then $w_{0} \in \partial h_{\tau}(I)$. Observe that $\tilde{r}_{j} \leq$ $r_{j}, 1 \leq j \leq k$. Obviously there are three cases:

If $w_{0}$ is the tangent point of two adjacent circles $c_{j}^{\tau}, c_{j+1}^{\tau}$ for some $1 \leq j \leq k$, then simple computation shows that

$$
\left|\phi^{\prime}\left(w_{0}\right)\right| \leq\left(\frac{1}{r_{j}}+\frac{1}{r_{j+1}}\right) \cdot\left(\frac{1}{\tilde{r}_{j}}+\frac{1}{\tilde{r}_{j+1}}\right)^{-1} \leq 1 .
$$

If $w_{0}$ is the intersecting point of two adjacent circles $c_{j}^{\tau}, c_{j+1}^{\tau}$ with dihedral angle $\theta_{j}=\Theta\left(\left[v_{j}, v_{j+1}\right]\right)>0$, then

$$
\left|\phi^{\prime}\left(w_{0}\right)\right| \leq \tilde{r}_{j} / r_{j} \leq 1
$$

If $w_{0}$ is not the intersecting point of two adjacent circles, then we also have

$$
\left|\phi^{\prime}\left(w_{0}\right)\right| \leq \tilde{r}_{j} / r_{j} \leq 1 \text {. }
$$

From (33), (34) and (35), it follows that $\left|\phi^{\prime}(w)\right| \leq 1$ holds on the closure of the region $h_{\tau}(I)$. Moreover, if $w \in h_{\tau}\left(\gamma_{j}\right)$ and $w$ is not an end point of the arc $h_{\tau}\left(\gamma_{i}\right), 1 \leq j \leq k$, then the strong maximal principle 3 implies that

$$
\left|\phi^{\prime}(w)\right|<1 \text {. }
$$

\footnotetext{
${ }^{3}$ The strong maximal principle states that: if a non-constant bounded harmonic function attains its minimal (resp. maximal) at $z_{0} \in \partial \Omega$, and the boundary $\partial \Omega$ satisfies an interior sphere
} 
Let $A_{j}$ (resp. $\left.\tilde{A}_{j}\right), 1 \leq j \leq k$, be the Euclidean center of the circle $c_{j}^{\tau}$ (resp. $\tilde{c}_{j}^{\tau}$ ). Denote by $B_{j}$ the vertex of the interstice $h_{\tau}(I)$ which is an intersecting point of $c_{j}^{\tau}$ and $c_{j+1}^{\tau}$ (see Figure 5 ). Similarly we denote by $\tilde{B}_{j}$ the corresponding vertex of the region $\tilde{h}_{\tau}(I)$.

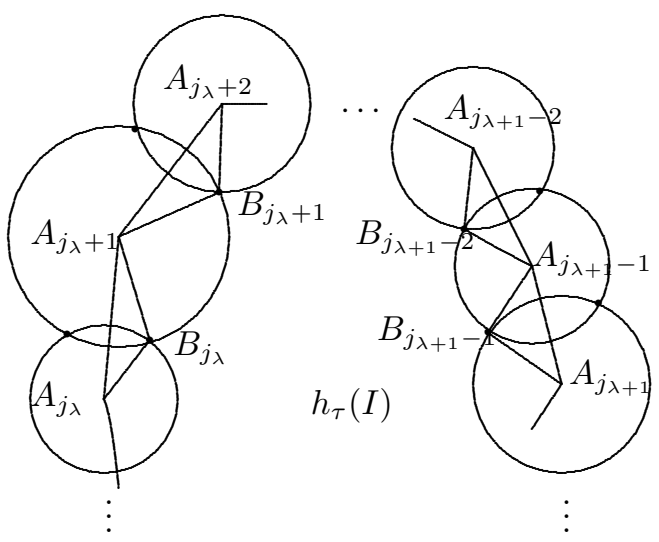

Figure 5

First we assume that $\tilde{r}_{j_{\lambda}}<r_{j_{\lambda}}, 1 \leq \lambda \leq \mu$, and $\tilde{r}_{j}=r_{j}, j \in\{1,2, \cdots, k\}$ $\backslash\left\{j_{1}, j_{2}, \cdots, j_{\mu}\right\}$. For any $1 \leq \lambda \leq \mu$, we consider the circles chain

$$
\left\{c_{j_{\lambda}}^{\tau}, c_{j_{\lambda}+1}^{\tau}, c_{j_{\lambda}+2}^{\tau}, \cdots, c_{j_{\lambda+1}-2}^{\tau}, c_{j_{\lambda+1}-1}^{\tau}, c_{j_{\lambda+1}}^{\tau}\right\}
$$

where $c_{j_{\mu+1}}^{\tau} \equiv c_{j_{1}}^{\tau}$. Then their radii satisfy

$$
r_{j}=\tilde{r}_{j}, \quad j_{\lambda}<j<j_{\lambda+1}, \text { and } \tilde{r}_{j_{\lambda}}<r_{j_{\lambda}}, \quad \tilde{r}_{j_{\lambda+1}}<r_{j_{\lambda+1}} .
$$

Therefore

$$
\begin{gathered}
\angle \tilde{A}_{j_{\lambda}} \tilde{A}_{j_{\lambda}+1} \tilde{B}_{j_{\lambda}} \leq \angle A_{j_{\lambda}} A_{j_{\lambda}+1} B_{j_{\lambda}} \\
\angle \tilde{A}_{j_{\lambda+1}} \tilde{A}_{j_{\lambda+1}-1} \tilde{B}_{j_{\lambda+1}-1} \leq \angle A_{j_{\lambda+1}} A_{j_{\lambda+1}-1} B_{j_{\lambda+1}-1}
\end{gathered}
$$

Using (36) and integrating $\left|\phi^{\prime}(w)\right|$ over the circular arc $h_{\tau}\left(\gamma_{j}\right)$ gives $\angle \tilde{B}_{j-1} \tilde{A}_{j} \tilde{B}_{j}<$ $\angle B_{j-1} A_{j} B_{j}, j_{\lambda}<j<j_{\lambda+1}$. We have

$$
\sum_{j_{\lambda}<j<j_{\lambda+1}} \tilde{\alpha}_{j}<\sum_{j_{\lambda}<j<j_{\lambda+1}} \alpha_{j}
$$

Summing up (37) over all $1 \leq \lambda \leq \mu$, and using $\sum_{j=1}^{k} \tilde{\alpha}_{j}=\sum_{j=1}^{k} \alpha_{j}=(k-2) \cdot \pi$, we have proved that

$$
\sum_{\lambda=1}^{\mu} \tilde{\alpha}_{j_{\lambda}}>\sum_{\lambda=1}^{\mu} \alpha_{j_{\lambda}},
$$

which completes the proof in case $\tilde{r}_{j}=r_{j}, j \in\{1,2, \cdots, k\} \backslash\left\{j_{1}, j_{2}, \cdots, j_{\mu}\right\}$.

Let us assume that $\tilde{r}_{j_{\lambda}}<r_{j_{\lambda}}, 1 \leq \lambda \leq \mu$, and

$$
\tilde{r}_{j} \geq r_{j}, j \in\{1,2, \cdots, k\} \backslash\left\{j_{1}, j_{2}, \cdots, j_{\mu}\right\} .
$$

condition at $z_{0}$, then the outer normal derivative of $u$ at $z_{0}$, if it exists, satisfies the strict inequality $\frac{\partial u}{\partial n}\left(z_{0}\right)>0 \quad$ (resp. <0); see e.g. Lemma 3.4 of [5]. 
Define a new positive numbers set $\left\{R_{j}\right\}_{1 \leq j \leq k}$ by setting $R_{j} \equiv r_{j}$ if $j \in\left\{j_{1}, j_{2}, \cdots\right.$, $j_{\mu}$ \}. Otherwise, set $R_{j} \equiv \tilde{r}_{j}$. Using the complex structure $[\tau: I \rightarrow \hat{\mathbb{C}}] \in \mathcal{T}_{I}$ and the radii $\left\{R_{j}>0\right\}_{1 \leq j \leq k}$, it follows from Lemma 3.3 that there exists an immersion $H_{\tau}: I \rightarrow \mathbb{C}$ such that $H_{\tau}\left(\gamma_{j}\right)$ is a circular arc on a circle $C_{j}^{\tau}$ with radius $R_{j}$, and the intersecting angle between the adjacent circles $C_{j}^{\tau}, C_{j+1}^{\tau}$ is $\theta_{j}, 1 \leq j \leq k$. Write $\vartheta_{j}, 1 \leq j \leq k$, the angles of the polygon by connecting the centers of the adjacent circles $C_{j}^{\tau}, C_{j+1}^{\tau}$ with straight arcs. As in the first part of this proof, it follows that

$$
\sum_{\lambda=1}^{\mu} \tilde{\alpha}_{j_{\lambda}}>\sum_{\lambda=1}^{\mu} \vartheta_{j_{\lambda}} .
$$

Similarly, by applying the same argument to $h_{\tau}$ and $H_{\tau}$,

$$
\sum_{j \notin\left\{j_{1}, j_{2}, \cdots, j_{\mu}\right\}} \alpha_{j} \geq \sum_{j \notin\left\{j_{1}, j_{2}, \cdots, j_{\mu}\right\}} \vartheta_{j} .
$$

With $\sum_{j=1}^{k} \alpha_{j}=\sum_{j=1}^{k} \vartheta_{j}=(k-2) \cdot \pi$, it follows from (39) that

$$
\sum_{\lambda=1}^{\mu} \alpha_{j_{\lambda}} \leq \sum_{\lambda=1}^{\mu} \vartheta_{j_{\lambda}} .
$$

Combining (38) with (40) gives

$$
\sum_{\lambda=1}^{\mu} \tilde{\alpha}_{j_{\lambda}}>\sum_{\lambda=1}^{\mu} \alpha_{j_{\lambda}},
$$

which completes the proof of Lemma 3.5

\section{Injection ANd SURJECTION of the map $H$. Part 2}

When there are no triangles in $\left\{\mathcal{F}_{1}, \mathcal{F}_{2}, \cdots, \mathcal{F}_{p}\right\}$, in this section we give the proof of the injection and surjection parts of Theorem 1.3 .

To prove the surjective part, recall that $P$ is the fixed circle pattern with the graph $G$ and $\left\{I_{1}, I_{2}, \cdots, I_{p}\right\}$ are the interstices of $P$. For any interstice $I_{i} \subset$ $\left\{I_{1}, I_{2}, \cdots, I_{p}\right\}$ with $k(>3)$ circular arcs $\left\{\gamma_{j}\right\}$ on its boundary, let $P\left(v_{i 1}\right)$; $P\left(v_{i 2}\right) ; \cdots ; P\left(v_{i k}\right)$ be the corresponding circles in $P$ adjacent to $I_{i}$ such that $\gamma_{j}=$ $\partial I_{i} \cap P\left(v_{i j}\right) ; 1 \leq j \leq k$.

The proof is similar to that of Lemma 3.3 in Section 3.5. For the given complex structure $\left[\tau: I_{i} \rightarrow \hat{\mathbb{C}}\right]$, we assume that $\tau\left(I_{i}\right) \subset \hat{\mathbb{C}}$ is a bounded domain in $\mathbb{C}$. Lay down a regular hexagonal packing of circles in $\mathbb{C}$, say each of radius $1 / n$. We obtain a circle packing $Q_{i n}$ which consists of all the circles intersecting the closed region $\tau\left(I_{i}\right)$. Assume that any boundary vertices of $\tau\left(I_{i}\right)$ lie inside of some boundary circles of $Q_{i n}$. Denote by $K_{i n}$ the contact graph of $Q_{i n}$. By using the contact graph $G$ and $K_{\text {in }}$ of $Q_{i n}, 1 \leq i \leq p$, we can construct a contact graph of $\hat{\mathbb{C}}$ by adding edges from the vertex $v_{i j}$ to the boundary vertices $\left\{v \in \partial K_{i n}: Q_{i n}(v) \cap \tau\left(\gamma_{j}\right) \neq \emptyset\right\}$ and adding edges between $v_{i j}$ and $v_{i(j+1)}, 1 \leq j \leq k$. The resulting graph $G_{n}$ is isomorphic to the 1-skeleton of a triangulation of $\hat{\mathbb{C}}$.

Let $E_{n}$ denote the edges set of $G_{n}$. Define a dihedral angle function $\Theta_{n}: E_{n} \rightarrow$ $[0, \pi / 2]$ by setting $\Theta_{n}(e)=\Theta(e)$ if $e=[v, w] \in E$. Otherwise set $\Theta_{n}(e)=0$. Since $G$ can be viewed as a subgraph of $G_{n}$, denote by the same notation $\left\{v_{1}, v_{2}, \cdots, v_{|V|}\right\}$ the vertices of $G_{n}$ associated with the vertices of $V$. 
Obviously $\left(G_{n}, \Theta_{n}\right)$ satisfies conditions $(i),(i i)$ and (iii) in Section.1. The result in the previous section implies that there is a circle pattern $P_{n}$ in $\hat{\mathbb{C}}$ realizing $\left(G_{n}, \Theta_{n}\right)$. It is unique up to Mobiüs transformations. We partially normalize this circle pattern such that the disk associated with $v_{1}$ is $D\left(v_{1}\right)=\hat{\mathbb{C}} \backslash\{|z|<1\}$. There is a correspondence of circles $c \rightarrow c^{\prime}$. Fixing a point $z_{0} \in \tau\left(I_{1}\right)$, let $c_{0}$ be a circle in $Q_{1 n}$ whose flower contains $z_{0}$. We further normalize the situation by a Mobiüs transformation which fixes the unit disk such that $c_{0}^{\prime}$ is centered at $0 \in\{|z| \leq 1\}$.

Denote by $F_{i n}^{\tau}$ the carrier of $Q_{i n}, 1 \leq i \leq p$. Then we have simplicial maps $\left\{A_{\text {in }}: F_{\text {in }}^{\tau} \rightarrow \mathbb{C}\right\}$ determined by the correspondence of centers of circles in the circle patterns $Q_{n}$ and $P_{n}$. By the Ring Lemma in [14], $A_{i n}: F_{i n}^{\tau} \rightarrow \mathbb{C}$ is an injective quasiconformal map. Letting $n \rightarrow \infty$, for $1 \leq i \leq p, A_{\text {in }}$ will converge to an analytic injective map $A_{i}^{\tau}: \tau\left(I_{i}\right) \rightarrow \mathbb{C}$ or a constant; see e.g. 14.

Suppose that $P_{n} \rightarrow P_{\infty}$. Then the following proposition implies that the circle pattern $P_{\infty}$ has the desired property.

Proposition 4.1. The circle pattern $P_{\infty}$ does not degenerate.

Proof. By contradiction, let us assume that the limit circle pattern $P_{\infty}$ degenerates. Denote by $W_{+}$the set of vertices $v$ in the graph $G$ for which $\lim _{n \rightarrow \infty} \rho\left(P_{n}(v)\right)>0$, and $W_{-}=V-W_{+}$. Note $v_{1} \in W_{+}$. Replacing by a subsequence if necessary, we may assume that for each $v \in W_{+}$, the sequence of circles $P_{n}(v)$ converges to some circle $P_{\infty}(v) \subset \mathbb{C}$.

First, suppose that $W_{-}=\emptyset$. That is, all circles in $P_{\infty}$ do not degenerate. Then at least one of the interstices, say $I_{1}^{\infty}$, degenerates. Hence $I_{1}^{\infty}$ degenerates to a point. Then all the boundary circles $\left\{P_{\infty}\left(v_{1 j}\right)\right\}_{1 \leq j \leq k}$ meet at a single point in $\mathbb{C}$. Since $k \geq 4$, the circles $P_{\infty}\left(v_{11}\right)$ and $P_{\infty}\left(v_{13}\right)$ are tangent. $P_{\infty}\left(v_{12}\right)$ and $P_{\infty}\left(v_{14}\right)$ are also tangent. Moreover $\Theta\left(\left[v_{1 j}, v_{1(j+1)}\right]\right)=\pi / 2,1 \leq j \leq 4$, which contradicts (ii) in Section 1.

Now suppose that $W_{-} \neq \emptyset$. If $\left|W_{+}\right| \leq 2$, then all interstices in $P_{\infty}$ degenerate. It is impossible by our normalization condition that the origin $0 \in\{|z| \leq 1\}$ lies in the interstice $I_{1}^{\tau}$.

From now on we assume that $\left|W_{+}\right| \geq 3$. Let $G_{\infty}$ denote the contact graph of $P_{\infty}$. If there are vertices $v, w \in G$ which do not share an edge in $G$ but they share an edge in the contact graph $G_{\infty}$ of $P_{\infty}$, then the circles $P_{\infty}(v)$ and $P_{\infty}(w)$ should then be tangent, since any interstice will degenerate to a point if it degenerates. $P_{\infty}(v)$ together with $P_{\infty}(w)$ cannot bound an interstice with any other circles in $P_{\infty}$. Therefore there exist $v^{\prime}, w^{\prime} \in G_{\infty}$ such that $w_{1} \equiv v, w_{2} \equiv v^{\prime}, w_{3} \equiv w, w_{4} \equiv w^{\prime}$ form a simple loop of four edges in the graph $G_{\infty}$. Furthermore $\Theta\left(\left[w_{j}, w_{j+1}\right]\right)=$ $\pi / 2,1 \leq j \leq 4$, which implies $\left[w_{j}, w_{j+1}\right] \in G$. Therefore $[v, w] \in G$, which is a contradiction.

If there are no new edges in the graph $G_{\infty}$, we can then view $G_{\infty}$ as a subgraph of $G$. Note that there are no triangles in $G$, which implies that there are no triangles in $G_{\infty}$. The subgraph $G_{\infty}$ divides the Riemann sphere into several connected domains $\left\{\mathcal{F}_{i}^{\infty}\right\}\left(1 \leq i \leq p^{\prime}\right)$. There exists a domain $\mathcal{F}_{i}^{\infty}$ such that

$$
\mathcal{F}_{i}^{\infty}=\mathcal{F}_{i_{1}} \cup \mathcal{F}_{i_{1}} \cdots \cup \mathcal{F}_{i_{q}}
$$

for some $p \geq q>1$, where $\left\{\mathcal{F}_{i_{j}}\right\} \subset\left\{\mathcal{F}_{i}\right\}_{1 \leq i \leq p}$. Each interstice $I_{i_{j}}^{\infty}, 1 \leq j \leq q$, of $P_{\infty}$ degenerates to a point, which implies that the interstice $I_{i}^{\infty}$ degenerates to a

\footnotetext{
${ }^{4}$ The flower centered at $c$ is the closed set consisting of $c$ and its interior, all circles tangent to $c$ and their interiors, and the interiors of all the triangular interstices formed by these circles.
} 
point. Therefore all boundary circles of $I_{i}^{\infty}$ must pass through a single point. It contradicts our assumption that there are no new edges in $G_{\infty}$.

To prove the injectivity of the map $H: \mathcal{D}_{P} \rightarrow \prod_{i=1}^{p} \mathcal{T}_{I_{i}}$, by contradiction, let us assume that there are circle patterns $Q, \tilde{Q}$ on $\hat{\mathbb{C}}$ such that $H(Q)=H(\tilde{Q})$. That is, there are holomorphic homeomorphisms $\phi_{i}: I_{i} \rightarrow \tilde{I}_{i}, 1 \leq i \leq p$, between the pairs of corresponding interstices, which map circular arcs or circles on $\partial I_{i}$ to the corresponding circular arcs or circles on $\partial \tilde{I}_{i}$.

Our goal is to prove that $\phi_{i}=\left.T\right|_{I_{i}}$ for some Mobiüs transformation $T: \hat{\mathbb{C}} \rightarrow \hat{\mathbb{C}}$ independent of $1 \leq i \leq p$. By using a Mobiüs transformation, we may assume $\infty \in I_{p}$ is a fixed point of $\phi_{p}: I_{p} \rightarrow \tilde{I}_{p}$. Then $\phi_{p}$ has the form

$$
\phi_{p}(z)=z+\frac{c_{1}}{z}+\frac{c_{2}}{z^{2}}+\cdots
$$

near $\infty$. If $\left|\phi_{i}^{\prime}(z)\right| \equiv 1$ for all $z \in I_{i}, 1 \leq i \leq p$, we deduce that

$$
\phi_{i}(z) \equiv z, z \in I_{i}
$$

Otherwise, there is at least some $z \in\left\{I_{i}\right\}$ such that $\left|\phi_{i}^{\prime}(z)\right| \neq 1$. Supposing $\left|\phi_{i}^{\prime}(z)\right|<$ 1 , then

$$
\min \left\{\log \left|\phi_{1}^{\prime}\right|, \log \left|\phi_{2}^{\prime}\right|, \cdots, \log \left|\phi_{p}^{\prime}\right|\right\}<0 .
$$

Hence there is some $\phi_{i}$, say $\phi_{1}$, such that

$$
\log \left|\phi_{1}^{\prime}\left(z_{0}\right)\right|=\min \left\{\log \left|\phi_{1}^{\prime}\right|, \log \left|\phi_{2}^{\prime}\right|, \cdots, \log \left|\phi_{p}^{\prime}\right|\right\}<0 .
$$

The maximal principle immediately implies that $z_{0} \in \partial I_{1}$. Let $v_{1}, v_{2}, \cdots, v_{k}$ be the vertices of $\mathcal{F}_{1}$. Write $\partial I_{1}=\bigcup_{j=1}^{k} \gamma_{j}$, where $\gamma_{j}=\partial I_{1} \cap Q\left(v_{j}\right)$ and write $\phi_{1}\left(\gamma_{j}\right)=$ $\partial \tilde{I}_{1} \cap \tilde{Q}\left(v_{j}\right), 1 \leq j \leq k$.

Suppose that $z_{0} \in \gamma_{j_{0}}$ for some $1 \leq j_{0} \leq k$. We assume that $z_{0}$ is not an end point of the circular arc $\gamma_{j_{0}}$ (otherwise, we can apply (33) or (34) to obtain the same results). Denote $r_{j} \equiv \rho\left(Q\left(v_{j}\right)\right)$ (resp. $\left.\tilde{r}_{j} \equiv \rho\left(\tilde{Q}\left(v_{j}\right)\right)\right), 1 \leq j \leq k$. Then the harmonic map $\log \left|\phi_{1}^{\prime}(z)\right|$ satisfies the Dirichlet-Neumann condition

$$
0 \geq \frac{\partial}{\partial n} \log \left|\phi_{1}^{\prime}\left(z_{0}\right)\right|=\frac{1}{r_{j_{0}}}-\frac{\left|\phi^{\prime}\left(z_{0}\right)\right|}{\tilde{r}_{j_{0}}},
$$

where the operator $\partial / \partial n$ denotes differentiation with respect to the exterior normal on $\gamma_{j_{0}}$. Therefore $\tilde{r}_{j_{0}} / r_{j_{0}} \leq\left|\phi_{1}^{\prime}\left(z_{0}\right)\right|<1$. It follows from (43) that, for $1 \leq i \leq p$,

$$
\tilde{r}_{j_{0}} / r_{j_{0}} \leq\left|\phi_{i}^{\prime}(z)\right|, \quad z \in I_{i} .
$$

If the circle $Q\left(v_{j_{0}}\right)$ is tangent to all other circles in the pattern $Q$, then (44) gives

$$
2 \pi \cdot \tilde{r}_{j_{0}}=\int_{Q\left(v_{j_{0}}\right)}\left|\phi_{1}^{\prime}(z)\right||d z| \geq \int_{Q\left(v_{j_{0}}\right)} \frac{\tilde{r}_{j_{0}}}{r_{j_{0}}}|d z|=2 \pi \cdot \tilde{r}_{j_{0}}
$$

which implies that $\left|\phi_{1}^{\prime}(z)\right| \equiv \tilde{r}_{j_{0}} / r_{j_{0}}, z \in Q\left(v_{j_{0}}\right)$. Therefore

$$
\frac{\partial}{\partial n} \log \left|\phi_{1}^{\prime}(z)\right|=0, \quad \forall z \in Q\left(v_{j_{0}}\right) .
$$

The strong maximal principle implies

$$
\log \left|\phi_{1}^{\prime}(z)\right| \equiv \tilde{r}_{j_{0}} / r_{j_{0}}, \forall z \in I_{1} .
$$

If $Q\left(v_{j_{0}}\right)$ is not isolated in $Q$, then $Q\left(v_{j_{0}}\right)$ belongs to a loop of circles or a chain of circles. For notational simplicity we assume that $Q\left(v_{j_{0}}\right)$ lies in a loop of circles 
$Q\left(v_{1}\right), Q\left(v_{2}\right), \cdots, Q\left(v_{k}\right), Q\left(v_{k+1}\right)=Q\left(v_{1}\right)$. If $Q\left(v_{j_{0}}\right)$ lies in a chain of circles, the proof is essentially the same and we omit it.

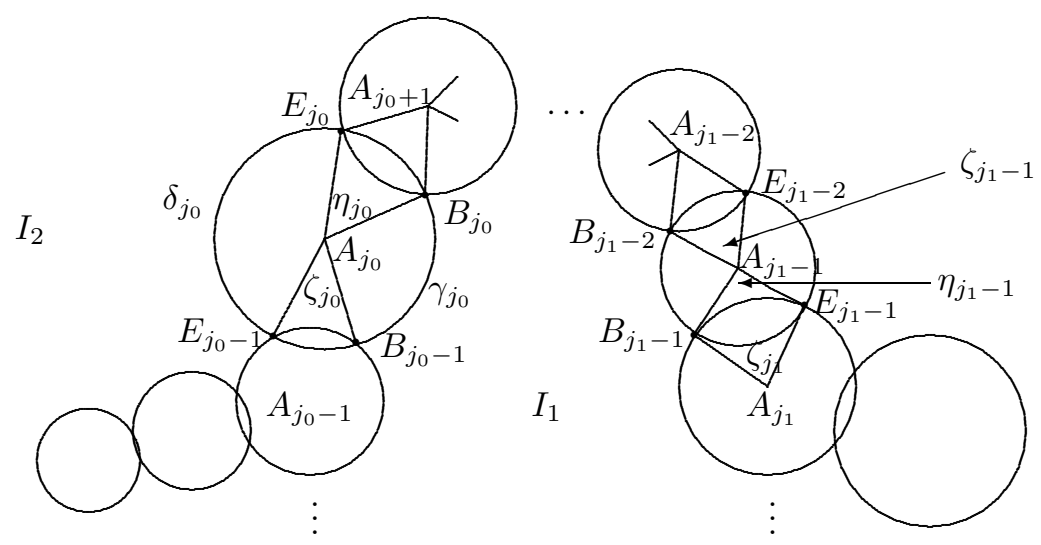

Figure 6

For $1 \leq j \leq k$, let $A_{j}$ denote the center of $Q\left(v_{j}\right)$ and let $B_{j}, E_{j}$ denote the intersecting points of $Q\left(v_{j}\right)$ and $Q\left(v_{j+1}\right)$. Let $\delta_{j}$ denote the circular arc between $E_{j-1}$ and $E_{j}$; see Figure 6 . Denote by $\tilde{A}_{j}, \tilde{B}_{j}, \tilde{E}_{j}$ the corresponding points in $\tilde{Q}$ and denote by $\tilde{\delta}_{j}$ the corresponding $\operatorname{arcs}$ in $\tilde{Q}$. If $\left|\phi_{1}^{\prime}(z)\right|$ is not a constant in the interstice $I_{1}$, from (44) and the strong maximal principle, then it follows that the length of the circular arc

$$
L\left(\tilde{\gamma}_{j_{0}}\right)=\int_{\gamma_{j_{0}}}\left|\phi_{1}^{\prime}(z)\right||d z|>\frac{\tilde{r}_{j_{0}}}{r_{j_{0}}} \cdot L\left(\gamma_{j_{0}}\right) .
$$

On the other hand, it follows from (43) that $L\left(\tilde{\delta}_{j_{0}}\right) \geq \frac{\tilde{r}_{j_{0}}}{r_{j_{0}}} \cdot L\left(\delta_{j_{0}}\right)$, where $\left\{\delta_{j_{0}}\right\}$ are all other boundary curves on the circle $Q\left(v_{j_{0}}\right)$ adjacent to some interstices. Therefore,

$$
\angle B_{j_{0}-1} A_{j_{0}} B_{j_{0}}<\angle \tilde{B}_{j_{0}-1} \tilde{A}_{j_{0}} \tilde{B}_{j_{0}} .
$$

For all other boundary curves $\delta_{j_{0}}$, (44) implies that $\left|\phi_{2}^{\prime}(z)\right| \geq \tilde{r}_{j_{0}} / r_{j_{0}}, \forall z \in \delta_{j_{0}}$. Hence

$$
\angle E_{j_{0}-1} A_{j_{0}} E_{j_{0}} \leq \angle \tilde{E}_{j_{0}-1} \tilde{A}_{j_{0}} \tilde{E}_{j_{0}} .
$$

For notational simplicity we assume that there are only two circles $Q\left(v_{j_{0}-1}\right)$ and $Q\left(v_{j_{0}+1}\right)$ adjacent to $Q\left(v_{j_{0}}\right)$. For $1 \leq j \leq k$, let $\eta_{j}$ (resp. $\left.\tilde{\eta}_{j}\right)$ denote the angle $\angle B_{j} A_{j} E_{j}$ (resp. $\angle \tilde{B}_{j} \tilde{A}_{j} \tilde{E}_{j}$ ), and let $\zeta_{j}$ (resp. $\tilde{\zeta}_{j}$ ) denote the angle $\angle B_{j-1} A_{j} E_{j-1}$ (resp. $\angle \tilde{B}_{j-1} \tilde{A}_{j} \tilde{E}_{j-1}$ ). From (48) and (49), it follows that $\tilde{\eta}_{j_{0}}<\eta_{j_{0}}$ or $\tilde{\zeta}_{j_{0}}<\zeta_{j_{0}}$. Assuming

$$
\tilde{\eta}_{j_{0}}<\eta_{j_{0}}
$$

and using $\Theta_{Q}\left(\left[v_{j_{0}}, v_{j_{0}+1}\right]\right)=\Theta_{\tilde{Q}}\left(\left[v_{j_{0}}, v_{j_{0}+1}\right]\right)$, we now obtain $\tilde{r}_{j_{0}+1} / r_{j_{0}+1}<\tilde{r}_{j_{0}} / r_{j_{0}}$, which proves

$$
G_{1} \equiv\left\{v: \rho(\tilde{Q}(v)) / \rho(Q(v))<\tilde{r}_{j_{0}} / r_{j_{0}}\right\} \neq \emptyset
$$


Without loss of generality, let the chain $\left\{v_{j_{0}+1}, \cdots, v_{j_{1}-1}\right\}$ be the maximal connected subgraph of $G_{1}$ containing $v_{j_{0}+1}$. Then their radii satisfy

$$
\frac{\tilde{r}_{j_{0}}}{r_{j_{0}}}>\frac{\tilde{r}_{j_{0}+1}}{r_{j_{0}+1}}, \frac{\tilde{r}_{j_{0}+2}}{r_{j_{0}+2}}, \cdots, \frac{\tilde{r}_{j_{1}-1}}{r_{j_{1}-1}} \text { and } \frac{\tilde{r}_{j_{0}}}{r_{j_{0}}} \leq \frac{\tilde{r}_{j_{1}}}{r_{j_{1}}} .
$$

Hence

$$
\tilde{\zeta}_{j_{1}} \leq \zeta_{j_{1}}
$$

Suppose that $\phi_{i_{j}}\left(\delta_{j}\right)=\tilde{\delta}_{j}$ for some $i_{j} \in\{1,2, \cdots, p\}$. Using (44) and integrating $\left|\phi_{i_{j}}^{\prime}\right|$ (resp. $\left.\left|\phi_{j_{j}}^{\prime}\right|\right)$ on the circular arc $\delta_{j}$ (resp. $\gamma_{j}$ ), we have thus proved that, for $j_{0}+1 \leq j \leq j_{1}-1$,

$$
\tilde{\eta}_{j}+\tilde{\zeta}_{j}<\eta_{j}+\zeta_{j}
$$

Therefore it follows from $\Theta_{Q}\left(\left[v_{j}, v_{j+1}\right]\right)=\Theta_{\tilde{Q}}\left(\left[v_{j}, v_{j+1}\right]\right), j_{0} \leq j \leq j_{1}$, that $\eta_{j_{0}}+$ $\zeta_{j_{1}}<\tilde{\eta}_{j_{0}}+\tilde{\zeta}_{j_{1}}$, which contradicts (50) and (51). Hence $\phi_{1}^{\prime}(z) \equiv c, \forall z \in I_{1}$ for some constant $c \in \mathbb{C}$. Therefore, " $\geq$ " should be "=" in (45), which contradicts (47). Hence $\phi_{i}^{\prime}(z) \equiv c$ for those $I_{i}$ adjacent to the circle $Q\left(v_{j_{0}}\right)$. By induction on all interstices $\left\{I_{i}\right\}$ and using the normalization (41), we conclude $\phi_{i}(z) \equiv z, \forall z \in$ $I_{i}, 1 \leq i \leq p$.

Therefore, there is a Mobiüs transformation $T: \hat{\mathbb{C}} \rightarrow \hat{\mathbb{C}}$ such that $T(Q)=\tilde{Q}$, which implies that $H: \mathcal{D}_{P} \rightarrow \prod_{i=1}^{p} \mathcal{T}_{I_{i}}$ is injective.

\section{Appendix: Some Classical Results.}

In order to obtain Lemmas 3.3 and 3.5 the following results are needed. These results are classical. Please refer to [15].

Suppose that $D, \tilde{D} \subset \mathbb{C}$ are domains bounded by circular arcs or circles $C_{j}, \tilde{C}_{j}$, where $j=1,2, \cdots, k$. Let $w=f(z): D \rightarrow \tilde{D}$ be a univalent conformal map which maps a circular arc or circle $C_{j} \subset \partial D$ onto a circular arc or circle $\tilde{C}_{j} \subset \partial D$. Denote by $C$ one of the boundary components bounded by $q$ circular arcs or circles $C_{j}, j=1,2, \cdots, q$, where $q \leq k$.

Suppose that $z=z(s)$ is the parametric representation of the boundary curves in terms of the arc length $s$. If $L_{j}$ is the length of the circular $\operatorname{arc} C_{j}$, then the variable $s$ will run from 0 to $L=\sum L_{j}$, and $z(s)$ will be an analytic function of $s$, except at the corners $s=L_{1}, L_{1}+L_{2}, \cdots$. We have the following Frenet formula for the curvature

$$
k(s)=\frac{1}{i} \frac{\ddot{z}}{\dot{z}}, \quad \dot{z}=\frac{d z}{d s} .
$$

By our assumption, the univalent conformal map $w=f(z)$ carries the curve system $\left\{C_{j}\right\}_{1 \leq j \leq k}$ into the system of circular $\operatorname{arcs}\left\{\tilde{C}_{j}\right\}_{1 \leq j \leq k}$ in the $w$-plane. We assume that $f(z)$ is analytic in the closed domain $\bar{D}=D \cup \partial D$, where $\partial D=\bigcup C_{j}$.

Again, the analytic curve system $\tilde{C}=\bigcup \tilde{C}_{j}$ bounds the domain $\tilde{D}$. Let $s^{\prime}$ be the arc length parameter on $\tilde{C}$. Since

$$
\frac{d \sigma}{d s}=\left|f^{\prime}(z)\right|, \quad z=z(s),
$$

we obtain that

$$
\frac{d w}{d \sigma}=f^{\prime}(z) \dot{z}\left|f^{\prime}(z)\right|^{-1}
$$


and hence by logarithmic differentiation with respect to the parameter $\sigma$ :

$$
\frac{d^{2} w}{d \sigma^{2}}\left(\frac{d w}{d \sigma}\right)^{-1}=\left[\frac{f^{\prime \prime}}{f^{\prime}} \dot{z}+\frac{\ddot{z}}{\dot{z}}-\frac{d}{d s} \log \left|f^{\prime}(z)\right|\right] \frac{d s}{d \sigma .}
$$

Comparing imaginary parts on both sides and using the formula (52) and its analogous for the domain $F$, we obtain that

$$
\tilde{k}(\sigma) d \sigma=k(s) d s+\Im\left\{d \log f^{\prime}(z)\right\},
$$

where $\tilde{k}(\sigma)$ denotes the curvature of the curve $\tilde{C}$ at the point $w(\sigma)$. Using (53) and the Cauchy-Riemann equations, we may bring (54) into the form

$$
\frac{\partial}{\partial n} \log \left|f^{\prime}(z)\right|=k(s)-\tilde{k}(\sigma)\left|f^{\prime}(z)\right|
$$

where the operator $\partial / \partial n$ denotes differentiation with respect to the exterior normal on the boundary curves $C$.

Since the boundary curve $C_{j}, 1 \leq j \leq k$, is a circular arc or circle, then $k(s)$ must be constant $1 / r_{j}$ on the curve $C_{j}$, where $r_{j}$ is the radius of the circle $C_{j}$. Similarly $\tilde{k}(\sigma)=1 / \tilde{r}_{j}$, where $\tilde{r}_{j}$ is the radius of $\tilde{C}_{j}$. Therefore, for $1 \leq j \leq k$,

$$
\frac{\partial}{\partial n} \log \left|f^{\prime}(z)\right|=\frac{1}{r_{j}}-\frac{\left|f^{\prime}(z)\right|}{\tilde{r}_{j}} .
$$

\section{ACKNOWLEDGEMENT}

The authors are very grateful to the anonymous referee for a careful reading of the manuscript and for many helpful suggestions.

\section{REFERENCES}

[1] L. Ahlfors, Conformal invariants, (McGraw-Hill, New York, 1973). MR0357743 (50:10211)

[2] A.F. Beardon \& K. Stephenson, 'The schwarz-Pick lemma for circle packings', Illinois. J. Math. 141 (1991) 577-606. MR1115988(93a:30028a)

[3] R. Brooks, 'On the deformation theory of classical Schottky groups', Duke. Math. J. 52, No 4, (1985) 1009-1024. MR816397 (87g:32024)

[4] P. G. Doyle, Z. X. He \& B. Rodin, 'Second derivatives of circle packings and conformal mappings', Discrete. \& Comput. Geom., 11 (1994) 35-49. MR:1244888 (94m:30017)

[5] D. Gilbarg \& N. S. Trudinger, Elliptic differential equations of second order (SpringerVerlag, Berlin, 1998).

[6] Z. X. He, 'Rigidity of infinite disk pattern', Ann. Math., 149 (1999) 1-33. MR.1680531 $(2000 \mathrm{j}: 30068)$

[7] Z. X. He \& O. Schramm, 'On the convergence of circle packing', Invent. Math., 125 (1996) 285-305. MR 1395721 (97i:30009)

[8] Z. X. He \& O. Schramm, 'The $C^{\infty}$ convergence of hexagonal disk packings to the Riemann map', Acta. Math., 180 (1998) 219-245. MR.1638772 (99j:52021)

[9] C. Hodgson, 'Deduction of Andreev' theorem from Rivin's characterization of convex hyperbolic polyhedra', Topology 90 (Columbus, OH, 1990), 185-193. MR1184410 (93h:57022)

[10] Y. Imayoshi \& M. Taniguchi, An introduction to Teichmüller spaces, (Springer-Verlag, Tokyo, 1992). MR1215481(94b:32031)

[11] O. Lehto. \& K. I. Virtanen., Quasiconformal Mappings in the plane, (Springer-Verlag, New York, 1973). MR0344463(49:9202)

[12] A. Marden \& B. Rodin, 'On Thurston's formulation and proof of Andreev's theorem', In Computational Methods and Function Theory, Lecture Notes in Mathematics 1435. Springer, (1989) 103-115. MR1071766 (92b:52040)

[13] I. A. Rivin. \& C. Hodgson, 'A characterization of compact convex polyhedra in hyperbolic 3-space', Invent. Math., 111 (1993) 77-111. 
[14] B. Rodin. \& D. Sullivan, 'The convergence of circle packings to the Riemann mapping', J. Diff. Geom., 26 (1987) 349-360. MR906396 (90c:30007)

[15] M. Schiffer. \& N. S. Hawley., 'Connections and conformal mappings', Acta. Math., 107 (1962) 175-274. MR0182720(32:202)

[16] O. Schramm, 'Rigidity of infinite (circle) packings', J. Amer. Math. Soc., 4 (1991) 127-149. MR1076089 (91k:52027)

[17] C. L. Siegel., Topics in complex function theory, Volume.1. (Wiley-Interscience, 1969).

[18] K. Stephenson, 'Circle packings in the approximation of conformal mappings', Bull. A.M.S., 23(1990) 407-415. MR 1049434 (92c:30009)

[19] W. Thurston, The geometry and topology of three manifolds, (Princeton University Press, 1977).

Institute of Mathematics, Academic of Mathematics and System Sciences, Chinese Academy of Sciences, Beijing 100190, People's Republic of China.

E-mail address: zhe00@earthlink.net

Current address: HUA Loo-Keng Key Laboratory of Mathematics, Chinese Academy of Sciences, Beijing 100190, People's Republic of China

Institute of Mathematics, Academic of Mathematics and System Sciences, Chinese Academy of Sciences, Beijing 100190, People's Republic of China.

E-mail address: liujsong@math.ac.cn

Current address: HUA Loo-Keng Key Laboratory of Mathematics, Chinese Academy of Sciences, Beijing 100190, People's Republic of China 